\title{
On a class of initial value problems and solitons for the KP equation: A numerical study
}

\author{
T. McDowell ${ }^{1}$, M. Osborne ${ }^{1}$, S. Chakravarty ${ }^{1 *}$, and Y. Kodama ${ }^{2}$ \\ ${ }^{1}$ Department of Mathematics, University of Colorado, Colorado Springs, CO 80918, USA \\ ${ }^{2}$ Department of Mathematics, Ohio State University, Columbus, OH 43210, USA
}

\begin{abstract}
Recent studies show that the Kadomtsev-Petviashvili (KP) equation admits a large class of exact solutions, referred to as the KP solitons, which are solitary waves localized along distinct rays and form web-like patterns in the $x y$-plane. It is also shown that each KP soliton can be uniquely parametrized by a specific element of the symmetric group. This paper is a numerical study of the initial value problem of the KP equation, for certain class of initial data which are not a small perturbation of any KP soliton. The numerical simulations demonstrate that the solution evolves to certain types of KP solitons whose permutations can be found from the initial data, as well as dispersive waves. In this sense, the KP scenario is analogous to its one-dimensional counterpart, namely, the Kortweg-deVries $(\mathrm{KdV})$ equation. The solution of the $\mathrm{KdV}$ equation is known to evolve asymptotically into sum of individual solitons and dispersive radiation. Although some numerical studies on KP were reported earlier in connection with the Mach reflection phenomena, the present study includes much larger class of initial data than those discussed in the previous studies.
\end{abstract}

Keywords: KP equation, KP solitons, chord diagrams

\section{Introduction}

An important example of physically interesting nonlinear wave equations was proposed in 1970 by Kadomtsev and Petviashvili [2] in their study of plasma waves. It is a $(2+1)$-dimensional, weakly nonlinear dispersive wave equation of the form

$$
\left(4 u_{t}+6 u u_{x}+u_{x x x}\right)_{x}+3 u_{y y}=0,
$$

where $x, y, t$ are the spatial coordinates and time; $u=u(x, y, t)$ represents the (normalized) wave amplitude; the subscripts denote partial derivatives. Equation (1) can be derived from the three-dimensional Euler equations for an irrotational and incompressible fluid under the assumptions that it describes the propagation of small amplitude, weakly dispersive, uni-directional waves with small, transverse variation (see e.g., $[1,11])$. In the literature, (1) is called the KP equation with negative dispersion, while the positive dispersion KP equation corresponds to (1) with a negative sign in front of the $u_{y y}$-term. Throughout this text, (1) will be simply referred to as the KP equation. From a physical perspective, the KP equation has been studied in the context of oblique interactions of ion-acoustic and shallow water solitary waves.

\footnotetext{
${ }^{*}$ Corresponding author. Email: chuck@ math.uccs.edu
} 
The KP equation (1) admits an important class of solitary wave solutions that are regular, non-decaying, and localized along distinct lines in the $x y$-plane. These are known as the KP solitons, which have been extensively studied in recent years leading to their complete classification using geometric and combinatorial techniques $[5,7,11]$. The KP solitons feature an arbitrary number of asymptotic line solitons in the far-field region $(|y| \gg 0)$ and a complex interaction pattern of intermediate solitons resembling a web-like structure in the near-field region. Several examples of the KP solitons have also been constructed in recent laboratory wave tank experiments demonstrating surface wave patterns generated by solitary wave interactions $[9,15]$.

The initial value problem of the KP equation plays an important role in the investigations of twodimensional wave patterns generated by the KP solitons. For its one-dimensional counterpart, namely the $\mathrm{KdV}$ equation, it is well known from theoretical considerations (see e.g., [1]) that rapidly decreasing (as $|x| \rightarrow \infty$ ), generic initial data evolves to solitons and dispersive waves that separate from the solitons. However, no such theory is currently available for the KP initial value problem with non-decaying initial data. In this paper, we conduct numerical studies of certain types of initial value problems in order to gain insight into the interaction properties of the KP solitons. Our numerical simulations reveal that the non-solitonic interactions of the initial data (which is viewed as a strong perturbation of the exact solution) generate dispersive waves and local spatial shifts along the soliton wave crest, while the resonant interactions create a wave pattern of intermediate solitons in the near-field region. The shifts along the soliton crests propagate toward the boundary along each asymptotic line soliton. Furthermore, the solution in the near-field region converges to a KP soliton inside a bounded domain $B$ moving with the wave pattern as the shifts along the soliton crests propagate out of the domain $B$. This convergence is in a local (or weighted) $\mathrm{L}^{2}$ sense over $B$. We provide a rough explanation of the convergence based on a combinatorial conjecture (see Section 3.1) about the minimal completion of chords associated with the asymptotic line solitons. This conjecture was first proposed in [12] where the authors considered the initial value problems with <-shape (sideways V) initial data that evolved to a special type of $(2,2)$-soliton solution of KP. The $(2,2)$-soliton solutions (see Section 2.2 for details) are KP solitons with a pair of asymptotic line solitons for $|y| \rightarrow \infty$, and there are seven distinct types of such solutions classified in [5, 7]. The work of [12] was further extended in [13] which systematically characterized all KP solitons arising from such $<-$ and Xshape. The numerical simulations of <-shape initial data was also treated recently in [8] using a different numerical scheme following Tanaka [16]. However, these works were incomplete in the sense that they found only a subset of the seven types of $(2,2)$-soliton solutions. In this paper, we consider wider varieties of initial data which evolve to the remaining types of $(2,2)$-solitons as well as other solutions not found previously. In our simulations, we choose initial data whose partial chord diagram encodes (via minimum completion) the permutation charecterizing the KP soliton that the data evolves to. The various other factors that mitigate the choice of initial data converging to a KP soliton are explained via illustrative numerical examples. The relations between the parameters defining the initial conditions and those of the exact solutions are also analyzed in details. In summary, the main highlights of this paper are as follows:

(a) The classification of KP solitons in [5, 7] predicts 7 distinct types of $(2,2)$-soliton solutions of KP. A natural question is to identify the class of initial data which give rise to each case of solutions. Previous investigations $[12,13,8]$, restricted to only $<-$ and $\mathrm{X}$-shape initial data, were not able to find all seven cases. In this paper, we study non-<-shape data which give rise to the missing cases, thereby exhausting all possible types of $(2,2)$-soliton solutions.

(b) In our numerical studies, we show that the initial data shed dispersive waves and evolve to exact KP solitons with a "kink" of the wave-crest due to local shift of the soliton center. Moreover, this shift propagates along the soliton wave-crest. To the best of our knowledge, this is the first time such behavior has been reported explicitly for KP solitons, or for (2+1)-dimensional soliton equations, in general.

(c) The choice of the class of initial data considered in this paper depends on the physical character- 
istics of the initial waveforms as well as certain combinatorial objects introduced in [5, 7] for the classification of KP solitons. A novel feature of this work is to combine those combinatorial ideas with with concrete numerical simulations.

The paper is organized as follows: Section 2 provides a brief summary of the theoretical development for the KP solitons and their classification obtained earlier in [5, 7, 11]. In Section 3, we describe our numerical simulations by considering initial data consisting of piece-wise defined line solitons joined together at the origin, representing interacting solitary waves. These initial conditions then evolve to various types of exact $(2,2)$-soliton solutions. A summary our work is provided in Section 4 as well as a brief outlook for future work. Finally, we have included two appendices. Appendix A provides additional details regarding the (2,2)-soliton solutions to aid the readers. Appendix B describes the numerical scheme used for our simulations and numerical tests to analyze the accuracy of the scheme. Preliminary results from a few of the numerical simulations presented in Section 3 were reported by some of the present authors in [8] but we carry out a detailed analysis of those simulations as well as present a number of new numerical results in our current work.

\section{Theoretical background}

The simplest example of a KP soliton is the one-soliton solution, which is a traveling wave of the form

$$
u(x, y, t)=\frac{1}{2}\left(k_{2}-k_{1}\right)^{2} \operatorname{sech}^{2} \frac{1}{2}\left(k_{2}-k_{1}\right)\left[x+\left(k_{1}+k_{2}\right) y-c_{12} t-x_{12}\right],
$$

and is localized along the line $L_{12}: x+\left(k_{1}+k_{2}\right) y-c_{12} t-x_{12}=0$ in the $x y$-plane for fixed $t$, as shown in Figure 1. The one-soliton is characterized by two real, distinct parameters $k_{1}<k_{2}$ which determine the soliton amplitude: $\frac{1}{2}\left(k_{2}-k_{1}\right)^{2}$, soliton speed: $c_{12}=k_{1}^{2}+k_{1} k_{2}+k_{2}^{2}$, and the soliton slope: $k_{1}+k_{2}=\tan \Psi_{12}$, where $\Psi_{12}$ is the angle, measured counterclockwise between the line $L$ and the $y$-axis. The soliton velocity is normal to the line $L_{12}$ and is given by

$$
V_{12}=\frac{c_{12}}{1+\left(k_{1}+k_{2}\right)^{2}}\left(1, k_{1}+k_{2}\right)
$$

We denote a one-soliton solution as the [1,2]-soliton since it is determined by the parameters $k_{1}$ and $k_{2}$.

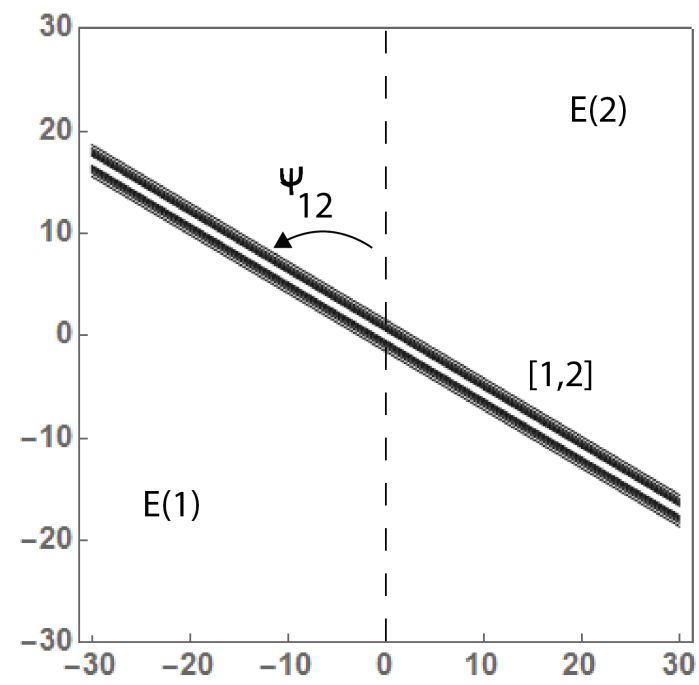

Figure 1: One-soliton solution of the KP equation 
The solution $u(x, y, t)$ of (1) is usually prescribed in terms of the $\tau$-function $\tau(x, y, t)$ as

$$
u(x, y, t)=2(\ln \tau)_{x x} .
$$

For the KP solitons, the corresponding $\tau$-function is a linear combination of exponential functions whose exponents are linear in $x, y$, and $t$. Importantly, $\tau(x, y, t)$ depends on

(i) $M$ distinct real parameters $\left\{k_{1}, k_{2}, \ldots, k_{M}\right\}$ ordered as $k_{1}<k_{2}<\cdots<k_{M}$, and

(ii) an $N \times M$ real matrix $A$ of full rank with $N<M$.

The explicit form of the $\tau$-function is given by the multi-sum

$$
\tau=\sum_{1 \leq j_{1}<j_{2}<\cdots<j_{N} \leq M} A\left(j_{1}, \ldots, j_{N}\right) E\left(j_{1}, \ldots, j_{N}\right), \quad E\left(j_{1}, \ldots, j_{N}\right):=\prod_{1 \leq r<s \leq N}\left(k_{j_{s}}-k_{j_{r}}\right) \prod_{l=1}^{N} E\left(j_{l}\right)
$$

where $E(j):=\exp \left(k_{j} x+k_{j}^{2} y-k_{j}^{3} t\right)$ and the maximal minor $A\left(j_{1}, \ldots, j_{N}\right)$ denotes the determinant of the $N \times N$ sub-matrix of $A$ with distinct columns labeled as $1 \leq j_{1}<j_{2}<\ldots<j_{N} \leq M$. The KP soliton solution $u(x, y, t)$ is non-singular for all $x, y$, and $t$ if and only if all maximal minors $A\left(j_{1}, \ldots, j_{N}\right)$ of the matrix $A$ are non-negative $[5,14]$. The corresponding matrix $A$ is called a totally non-negative (TNN) matrix. The other two properties of the $A$-matrix used in this paper are as follows:

(i) Without loss of generality, the $N \times M$ matrix $A$ can be taken in reduced row-echelon form (RREF) since elementary row operations change the $\tau$-function by a scale factor and leaves the solution in (3) invariant.

(ii) The $A$-matrix is irreducible. This means that each column of $A$ contains at least one nonzero element, and each row contains at least one nonzero element other than the pivot once $A$ is in RREF.

For example, the $\tau$-function for the one-soliton solution (2) is obtained by choosing $N=1$ and $M=2$ in (4). The $1 \times 2$ matrix $A=(1, a)$ with $a>0$ yields the $\tau$-function

$$
\tau(x, y, t)=A(1) E(1)+A(2) E(2)=E(1)+a E(2),
$$

which leads to (2) after using the formula in (3). The positive parameter $a$ is related to the $x$-intercept at $t=0$ of the line $L_{12}: x+\left(k_{1}+k_{2}\right) y-c_{12} t-x_{12}=0$ along which the soliton is localized, i.e., $x_{12}=$ $-\ln a /\left(k_{2}-k_{1}\right)$. Note from Figure 1 that the one-soliton solution is exponentially small in regions of the $x y$-plane where the exponentials $E(1)$ and $E(2)$ are dominant and is localized along the line $L_{12}$ where $E(1)$ and $E(2)$ are of the same order.

In earlier works $[4,5,7,11]$, it was shown that the general soliton solution given by equations (3) and (4) consists of $N$ asymptotic line solitons as $y \gg 0$ and $M-N$ asymptotic line solitons as $y \ll 0$. This solution is referred to as a $(M-N, N)$-soliton solution. Each asymptotic line soliton has the form of an exact 1-soliton solution (2), i.e., has the asymptotic form

$$
\frac{1}{2}\left(k_{j}-k_{i}\right)^{2} \operatorname{sech}^{2} \frac{1}{2}\left(k_{j}-k_{i}\right)\left[x+\left(k_{i}+k_{j}\right) y-c_{i j} t-x_{i j}\right],
$$

with $c_{i j}=k_{i}^{2}+k_{i} k_{j}+k_{j}^{2}$ and is uniquely parametrized by a pair of distinct $k$-parameters $k_{i}<k_{j}$ for $i<j$. Hence, we denote each asymptotic line soliton as the $[i, j]$-soliton in this article. Furthermore, the index pair $[i, j], i<j$ associated with an asymptotic line soliton is uniquely characterized by a fixed-point free permutation of the index set $[1,2, \ldots, M]$. It is given by a pairing map $\pi$ such that

(i) $\pi(i)=j, \quad$ if $[i, j]$ labels an asymptotic soliton for $y \gg 0$, and

(ii) $\pi(j)=i, \quad$ if $[i, j]$ labels an asymptotic soliton for $y \ll 0$. 

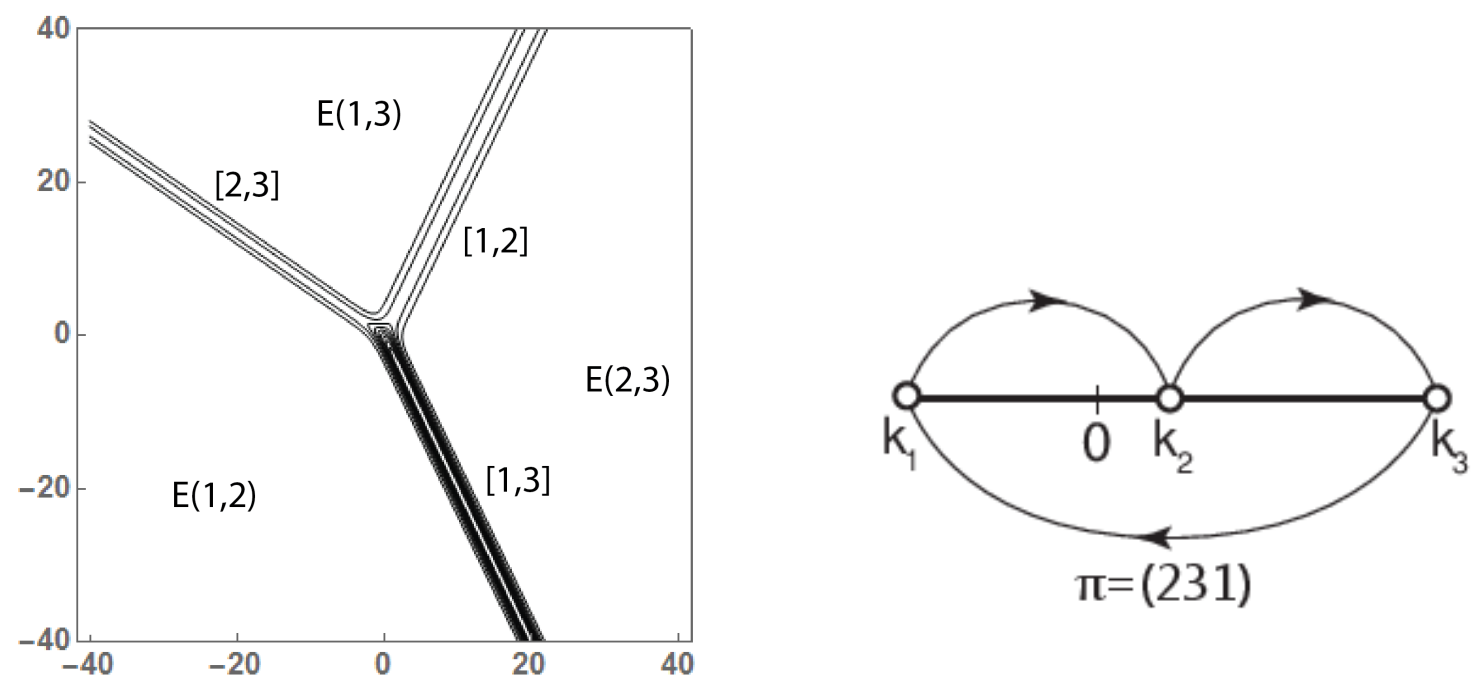

Figure 2: A $(1,2)$-soliton solution and its chord diagram. The $E(i, j)$ on each region indicates the dominant exponential term. For this figure, the $k$-parameters are $\left(k_{1}, k_{2}, k_{3}\right)=\left(-\frac{3}{4}, \frac{1}{4}, \frac{5}{4}\right)$ and the $A$-matrix parameters are $a=\frac{1}{2}$ and $b=1$.

The map $\pi$, also known as a derangement, can be conveniently represented by a chord diagram where the pairing between the indices are indicated by a chord. Specifically, a chord diagram consists of $M$ marked points on a line with $N$ directed chords above the line indicating the pairings $\pi(i)=j, i<j, i=1,2, \ldots, N$, and $M-N$ directed chords below the line indicating the pairings $\pi(j)=i, i<j, j=1,2, \ldots, M-N$. These chord diagrams are illustrated in each of the examples presented in the following subsections. Thus, each $(M-N, N)$-soliton solution (3) generated by the $\tau$-function (4) is represented by a chord diagram associated with the derangement $\pi$. Conversely, for each derangement $\pi$ there exists a class of $(M-N, N)$ soliton solutions whose asymptotic solitons are uniquely determined by the derangement $\pi$.

In the following subsections, we consider some elementary examples of exact $(M-N, N)$-soliton solutions of the KP equation. These examples illustrate the theory described above, as well as form the basis of our numerical work presented later in Section 3.

\subsection{The Y-solitons}

Let us consider the case $N=2, M=3$ in (4) with an ordered set of parameters $\left\{k_{1}, k_{2}, k_{3}\right\}$ together with a $2 \times 3$ matrix of the form

$$
A=\left(\begin{array}{ccc}
1 & 0 & -b \\
0 & 1 & a
\end{array}\right), \quad a>0, \quad b>0 .
$$

Note that $A$ above is a TNN matrix. From (4), the corresponding $\tau$-function is given by

$$
\tau=E(1,2)+a E(1,3)+b E(2,3)
$$

where $E(i, j)=\left(k_{j}-k_{i}\right) E(i) E(j)$ as defined in (4). Figure 2 shows a contour plot of the solution obtained from (3) after using the $\tau$-function from (4). It is a $(1,2)$-soliton in the terminology used above, also referred to as the Y-soliton. The region of the $x y$-plane where each exponential function $E(i, j)$ dominates is also indicated in the figure. The asymptotic line solitons are localized along the boundary of such adjacent regions. That is, the dominant exponential functions balance one another along the boundary. For $y \gg 0$, the boundary between the regions with the dominant exponentials $E(1,2)$ and $E(1,3)$ defines the $[2,3]$-soliton solution. This can be seen as follows: along this boundary the $\tau$-function can be approximated 

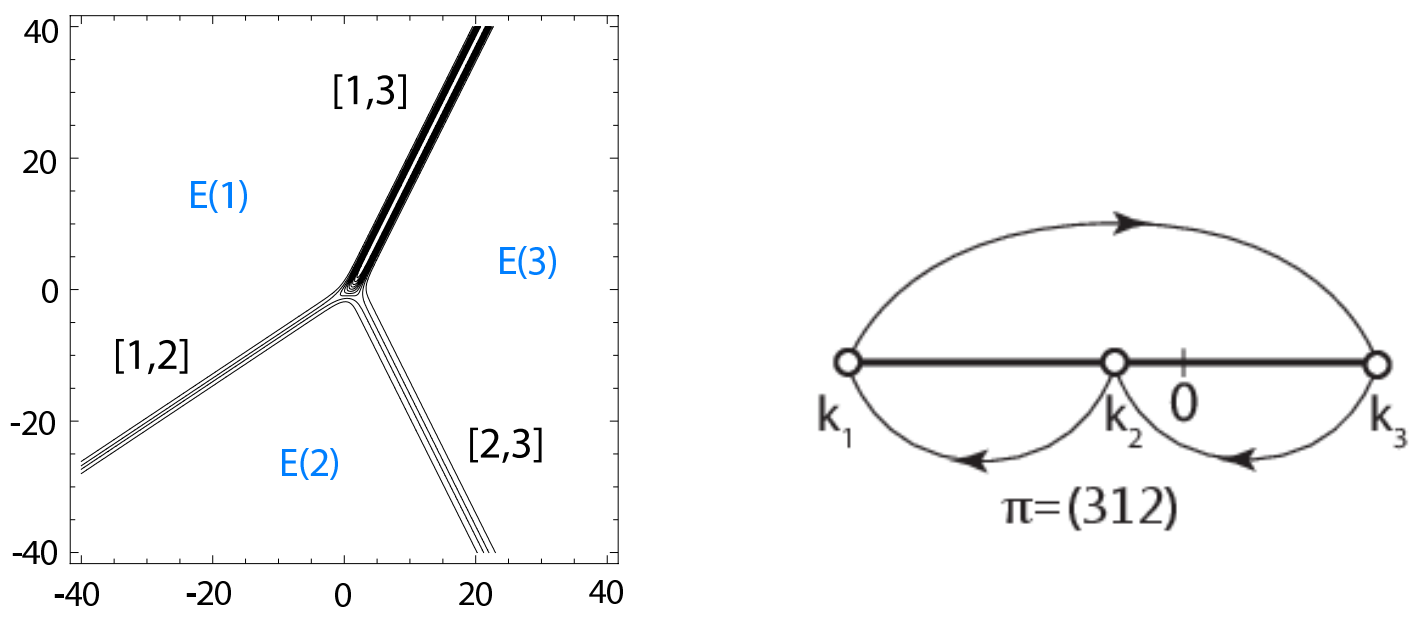

Figure 3: A $(2,1)$-soliton solution and its chord diagram. The $E(i)$ on each region indicates the dominant exponential term. For this figure, the $k$-parameters are $\left(k_{1}, k_{2}, k_{3}\right)=\left(-\frac{5}{4},-\frac{1}{4}, \frac{3}{4}\right)$ and the $A$-matrix parameters are $a=b=1$.

as $\tau \approx E(1,2)+a E(1,3)$ so that we have from (3) that

$$
u(x, y, t) \approx \frac{1}{2}\left(k_{3}-k_{2}\right)^{2} \operatorname{sech}^{2} \frac{1}{2}\left(k_{3}-k_{2}\right)\left[x+\left(k_{2}+k_{3}\right) y-c_{23} t-x_{23}\right],
$$

where $c_{23}$ and $x_{23}$ are given by

$$
c_{23}=k_{2}^{2}+k_{2} k_{3}+k_{3}^{2}, \quad x_{23}=\frac{1}{k_{3}-k_{2}}\left[\ln \frac{k_{2}-k_{1}}{k_{3}-k_{1}}-\ln a\right] .
$$

Similar analyses lead to the asymptotic [1,2]-soliton for $y \gg 0$ and the asymptotic $[1,3]$-soliton for $y \ll 0$ with $c_{i j}=k_{i}^{2}+k_{i} k_{j}+k_{j}^{2}$, and

$$
x_{12}=\frac{1}{k_{2}-k_{1}}\left[\ln \frac{k_{3}-k_{1}}{k_{3}-k_{2}}-\ln \frac{b}{a}\right], \quad x_{13}=\frac{1}{k_{3}-k_{1}}\left[\ln \frac{k_{2}-k_{1}}{k_{3}-k_{2}}-\ln b\right] .
$$

The figure in the right panel of Figure 2 displays a chord diagram representing a permutation of the index set $\{1,2,3\}$. Note that in the diagram, the indices are labeled by the ordered parameters $k_{1}, k_{2}, k_{3}$ (instead of 1,2,3). The two up-chords from $k_{1}$ to $k_{2}$ and $k_{2}$ to $k_{3}$ denote the pairing maps $\pi(1)=2$ and $\pi(2)=3$, respectively. They represent the asymptotic line solitons $[1,2]$ and $[2,3]$ for $y \gg 0$. Similarly, the down-chord from $k_{3}$ to $k_{1}$ denotes the pairing map $\pi(3)=1$ which represents the asymptotic line soliton $[1,3]$ for $y \ll 0$. Thus, the chord diagram represents the derangement $\pi=(231)$. At the same time, it identifies the asymptotic line solitons which also tells us that this is a $(1,2)$-type soliton solution. Next, note that the values of the $k$-parameters are indicated on the horizontal line, i.e., $k_{1}<0<k_{2}<k_{3}$ in the chord diagram. Then it follows that the $[1,2]$-soliton has the smallest slope which is negative (oriented clockwise from the $y$-axis) since $k_{1}+k_{2}=\tan \Psi_{12}<0$. Similarly, the [2,3]-soliton has a positive slope (oriented counter-clockwise from the $y$-axis) since $k_{2}+k_{3}>0$. The [1,3]-soliton has the largest amplitude given by $\frac{1}{2}\left(k_{3}-k_{1}\right)^{2}$. Its slope is positive but smaller than the [2,3]-soliton. Thus, the amplitude and orientation informations of the asymptotic line solitons can be also gathered from the chord diagram.

An inverted version of the Y-soliton can be obtained by considering $N=1, M=3$ in (4) with an ordered set of parameters $\left\{k_{1}, k_{2}, k_{3}\right\}$ together with the $A$-matrix $A=\left(\begin{array}{lll}1 & a & b\end{array}\right), \quad a>0, \quad b>0$. The corresponding $\tau$-function is given by $\tau=E(1)+a E(2)+b E(3)$. The solution shown in Figure 3 is a $(2,1)$-soliton, also referred to as the $X$-soliton. It consists of a $[1,3]$-soliton for $y \gg 0$, and $[2,3]$ - and $[1,2]$-solitons for $y \ll 0$, represented by the derangement $\pi=(312)$ whose chord diagram is also shown 
in Figure 3. The $k$-parameters $\mathrm{n}$ this case are ordered as $k_{1}<k_{2}<0<k_{3}$ as shown in the chord diagram. This implies that the $[1,2]$-soliton has the smallest slope which is negative since $k_{1}+k_{2}=\tan \Psi_{12}<0$ and the $[2,3]$-soliton has a positive slope as $k_{2}+k_{3}>0$. The [1,3]-soliton has the largest amplitude given by $\frac{1}{2}\left(k_{3}-k_{1}\right)^{2}$. Its slope is negative but larger than the $[1,2]$-soliton. Each asymptotic soliton is of the form in (5) with

$$
x_{12}=-\frac{1}{k_{2}-k_{1}} \ln a, \quad x_{13}=-\frac{1}{k_{3}-k_{1}} \ln b, \quad x_{23}=\frac{1}{k_{3}-k_{2}} \ln \frac{a}{b} .
$$

The above expressions are derived from the balance of dominant exponentials $E(i)$ in the same fashion as in the Y-soliton case above.

The following information regarding Y-solitons will be important for our discussions of the numerical results presented in Section 3.

(a) The chord diagram plays an important role to represent as well as to characterize the constituent asymptotic line solitons of a general $(M-N, N)$-soliton solution of (1) besides representing the derangements $\pi$. The chord representations are used throughout this paper to denote initial data and the timeevolved solution in terms of incomplete chords and their completion (see Section 3.1 for details). In the general $(M-N, N)$-soliton case, one marks the ordered set of real distinct $k$-parameters $k_{1}, k_{2}, \ldots, k_{M}$ along a horizontal line, then connects $k_{i}$ and $k_{j}$ by an up-chord if the indices satisfy $\pi(i)=j$ or by a down-chord if $\pi(j)=i$ for $i<j$. The up- and down-chords represent a $[i, j]$-soliton for $y \gg 0$ and $y \ll 0$, respectively. Furthermore, the values of $k_{i}$ and $k_{j}$ indicate the amplitude and orientation of the $[i, j]$-soliton by $\frac{1}{2}\left(k_{j}-k_{i}\right)^{2}$ and $\tan \Psi_{i j}=k_{i}+k_{j}$ where $\Psi_{i j}$ denotes the angle between the $[i, j]$-soliton and the $y$-axis.

(b) The Y-soliton solution described in Section 2.1 is a resonant three-wave interaction of the asymptotic line solitons $[1,2],[2,3]$, and $[1,3]$ at the vertex of the Y-junction. Denoting the wave-vector and frequency of each $[i, j]$-soliton by $\mathbf{K}_{i j}=\left(k_{j}-k_{i}, k_{j}^{2}-k_{i}^{2}\right), \Omega_{i j}=k_{j}^{3}-k_{i}^{3}$, the resonant condition reads

$$
\mathbf{K}_{13}=\mathbf{K}_{12}+\mathbf{K}_{23}, \quad \Omega_{13}=\Omega_{12}+\Omega_{23} .
$$

In terms of the position shifts $x_{i j}$ of each $[i, j]$-soliton the resonance condition is also expressed as

$$
\left(k_{2}-k_{1}\right) x_{12}+\left(k_{3}-k_{2}\right) x_{23}=\left(k_{3}-k_{1}\right) x_{13},
$$

which is readily verified from the expressions for the $x_{i j}$ given above. The resonant vertex $\left(x_{0}(t), y_{0}(t)\right)$, which is the common intersection point of all three lines $L_{i j}: x+\left(k_{i}+k_{j}\right) y=c_{i j} t+x_{i j}, \quad 1 \leq i<j \leq 3$, is given by

$$
x_{0}(t)=-\left(k_{1} k_{2}+k_{1} k_{3}+k_{2} k_{3}\right) t+\frac{\left(k_{1}+k_{3}\right) x_{12}-\left(k_{1}+k_{2}\right) x_{13}}{k_{3}-k_{2}}, \quad y_{0}(t)=\left(k_{1}+k_{2}+k_{3}\right) t+\frac{x_{13}-x_{12}}{k_{3}-k_{2}} .
$$

In fact, it suffices to take the intersection of any two of the three lines; the resonant condition ensures that the third line passes through the same point. The above formulas will be useful in the analyses of the numerical simulations in Section 3.

\subsection{The (2,2)-solitons}

The (2,2)-solitons are KP solitons which consist of two asymptotic line solitons as $y \rightarrow \pm \infty$ according to the terminology introduced earlier in the section. The $\tau$-function for these solutions is obtained from (4) by considering all possible $2 \times 4 \mathrm{TNN}$, irreducible $A$-matrices and a set of 4 real parameters $\left\{k_{1}, k_{2}, k_{3}, k_{4}\right\}$. Thus, the explicit form of a $(2,2)$-soliton $\tau$-function is given by

$$
\tau(x, y, t)=\sum_{1 \leq i<j \leq 4} A(i, j) E(i, j)
$$




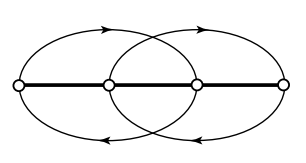

(3412)

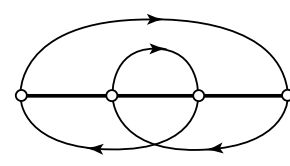

(4312)

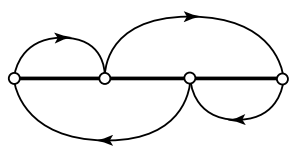

(2413)

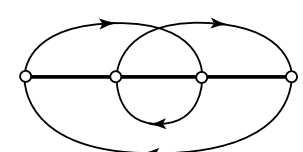

(3421)

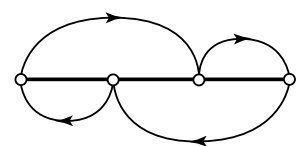

(3142)

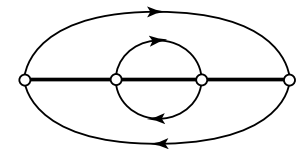

(4321)

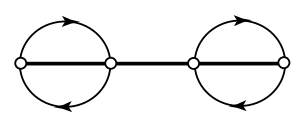

(2143)

Figure 4: The chord diagrams for seven different types of $(2,2)$-soliton solutions [7].
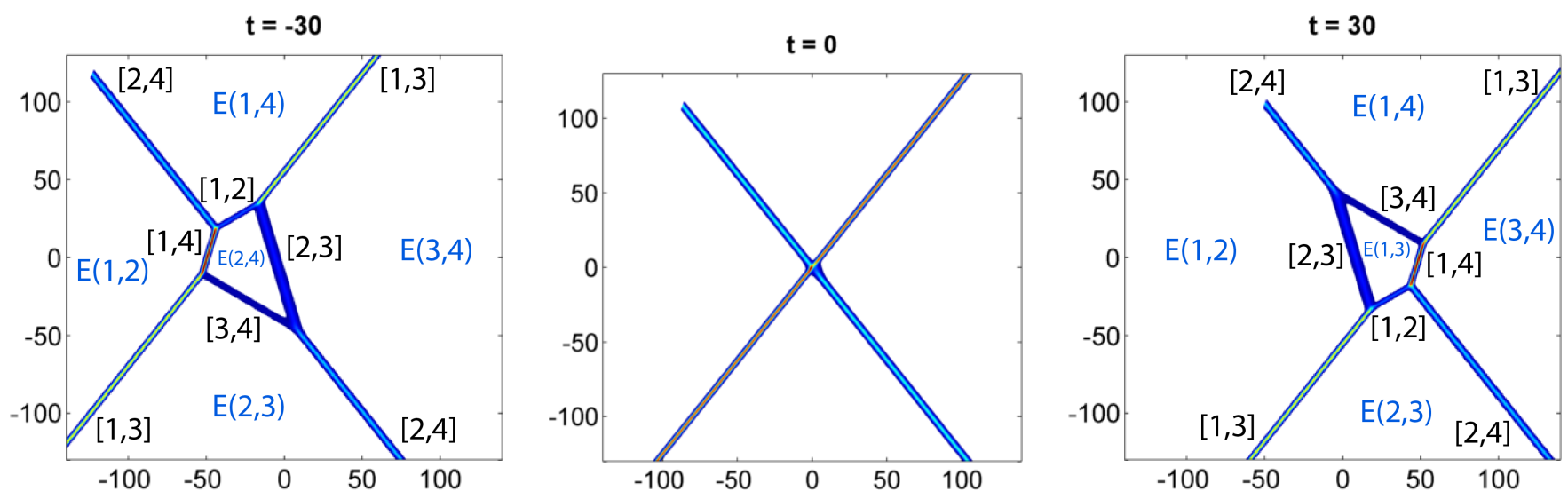

Figure 5: A (3412)-type soliton with $k$-parameters: $\{-1.4,-0.3,0.6,1.1\}$. The parameters in the $A$ matrix are chosen such that the $[1,3]$ - and $[2,4]$-solitons intersect at the origin at $t=0 . E(i, j)$ indicates the dominant exponentials in each region. Note that the dominant exponential inside the box switches from $E(2,4)$ for $t<0$ to $E(1,3)$ for $t>0$.

where $E(i, j)=\left(k_{j}-k_{i}\right) E(i) E(j)$ as given by (4) (with $N=2$ ) and $E(j):=\exp \left(k_{j} x+k_{j}^{2} y-k_{j}^{3} t\right)$. It is easy to verify that there are only two distinct types of RREF for $2 \times 4$, irreducible, TNN matrices, namely,

$$
\left(\begin{array}{cccc}
1 & 0 & -c & -d \\
0 & 1 & a & b
\end{array}\right) \quad \text { and } \quad\left(\begin{array}{cccc}
1 & a & 0 & -c \\
0 & 0 & 1 & b
\end{array}\right)
$$

where $a, b, c, d$ are nonnegative real parameters. For the first type of RREF, $a d \neq 0$. Then one can deduce that there are 5 possible cases when all the principal minors $A(i, j) \geq 0$. These are:
(1) $a d-b c>0, b c \neq 0$
(2) $a d-b c=0, b c \neq 0$
(3) $b=0, c \neq 0$,
(4) $c=0, b \neq 0$
$(5) b=c=0$.

For the second type of RRE, $a b \neq 0$, and there are only two cases:

$$
(1) c=0, \quad(2) c \neq 0 .
$$

Thus, there are a total of seven distinct cases of irreducible, TNN $A$-matrices, each giving rise to a different $(2,2)$-soliton solution of the KP equation via (3) and (9). The complete classification of the (2,2)-soliton solutions including the identification of the asymptotic line solitons at $|y| \rightarrow \infty$ and the pairing map $\pi$ can be found in Refs $[4,5]$. The pairing map $\pi$, in this case, is a fixed-point free permutation of four indices $\{1,2,3,4\}$. The associated chord diagrams are illustrated in Figure 4.

Example: We consider a specific example in order to illustrate the basic features of the $(2,2)$-soliton solutions. This case corresponds to $\pi=(3412)$. From its chord diagram in Figure 4, one can see that the 
asymptotic line solitons for this solution consist of a pair of [1,3]- and [2,4]-solitons as $|y| \rightarrow \infty$. These asymptotic solitons interact resonantly to generate four intermediate solitons identified as the $[1,2]-$, [2,3], $[1,4]$ - and $[3,4]$-solitons which form a box at the interaction region. Figure 5 depicts the time-evolution of a (3412)-type soliton. One can also observe that the [1,4]- and [2,3]-solitons forming the left and right edges of the box switch as time evolves from $t<0$ to $t>0$. The [1,2]- and [3,4]-solitons forming the top and bottom edges of the box are also swapped with time. This switch occurs because the dominant exponential function inside the box changes from being $E(2,4)$ when $t<0$ to $E(1,3)$ when $t>0$, whereas the dominant exponentials in the far-field regions do not change with time. The $A$-matrix corresponding to the (3412)-type soliton is given by

$$
\left(\begin{array}{cccc}
1 & 0 & -c & -d \\
0 & 1 & a & b
\end{array}\right), \quad a, b, c, d>0, \quad a d-b c>0 .
$$

The parameters $a, b, c, d$ in the $A$-matrix are related to the $x$-intercepts of the asymptotic and intermediate line solitons appearing in the (3412)-type soliton solution. For example, one obtains

$$
b=\frac{k_{2}-k_{1}}{k_{4}-k_{1}} e^{-\theta_{24}^{+}}, \quad c=\frac{k_{2}-k_{1}}{k_{3}-k_{2}} e^{-\theta_{13}^{-}}, \quad \frac{D}{b}=\frac{k_{4}-k_{1}}{k_{4}-k_{3}} e^{-\theta_{13}^{+}}, \quad d=\frac{k_{2}-k_{1}}{k_{4}-k_{2}} e^{-\theta_{14}^{*}},
$$

where $D=a d-b c, \theta_{i j}=\left(k_{j}-k_{i}\right) x_{i j}, x_{i j}$ denotes the $x$-intercept of the $[i, j]$-soliton, the superscripts \pm indicate the $[i, j]$-soliton appearing in $y>0$ or $y<0$, and $\theta_{14}^{*}$ denotes the intermediate [1,4]-soliton for $t<0$. The above relations were derived in [7] $]^{1}$ by considering the balance of the dominant exponential functions $E(i, j)$ at different regions of the $x y$-plane for a fixed value of $t$, as shown for the Y-soliton in Section 2.1.

The basic features of the remaining (2,2)-soliton solutions are summarized in Appendix A in Figures 21, 22 and in Table 1. The interested reader is also referred to the works $[5,7,11]$ for more details.

\section{Numerical simulations}

In this paper we carry out direct numerical simulations of the KP equation (1) in order to study the interaction properties of the KP solitons. Specifically, we show that the numerical solutions corresponding to certain types of initial conditions approach asymptotically in time to the exact solutions discussed in Section 2. We have developed MATLAB codes to run these numerical simulations with initial data that are non-decaying at the boundary of the numerical domain. In particular, the the initial waves are extended all the way to the boundary in the $y$-direction but their interactions take place in a finite region in the interior of the numerical domain and away from the boundary. The accuracy of our numerical codes is tested by comparing the numerical solution with the exact (3412)-type soliton. The numerical scheme and the error estimates with respect to various scheme parameters are discussed in Appendix B.

\subsection{Chord completion and convergence}

We outline below the procedure followed in our numerical simulations. We take a set of $k$-parameters to construct initial data obtained by gluing together two (more) semi-infinite asymptotic line solitons of the form given in (5) at $y=0$. This initial waveform corresponds to a "partial" chord diagram representing the initial asymptotic solitons as illustrated by the example shown in the left frame of Figure 6 . If the initial condition evolves to a KP soliton, the partial chord diagram is then completed by the additional chords.

\footnotetext{
${ }^{1}$ Our notation for $\theta_{i j}^{ \pm}$is different from [7] where \pm denotes asymptotic solitons for $x>0$ and $x<0$.
} 

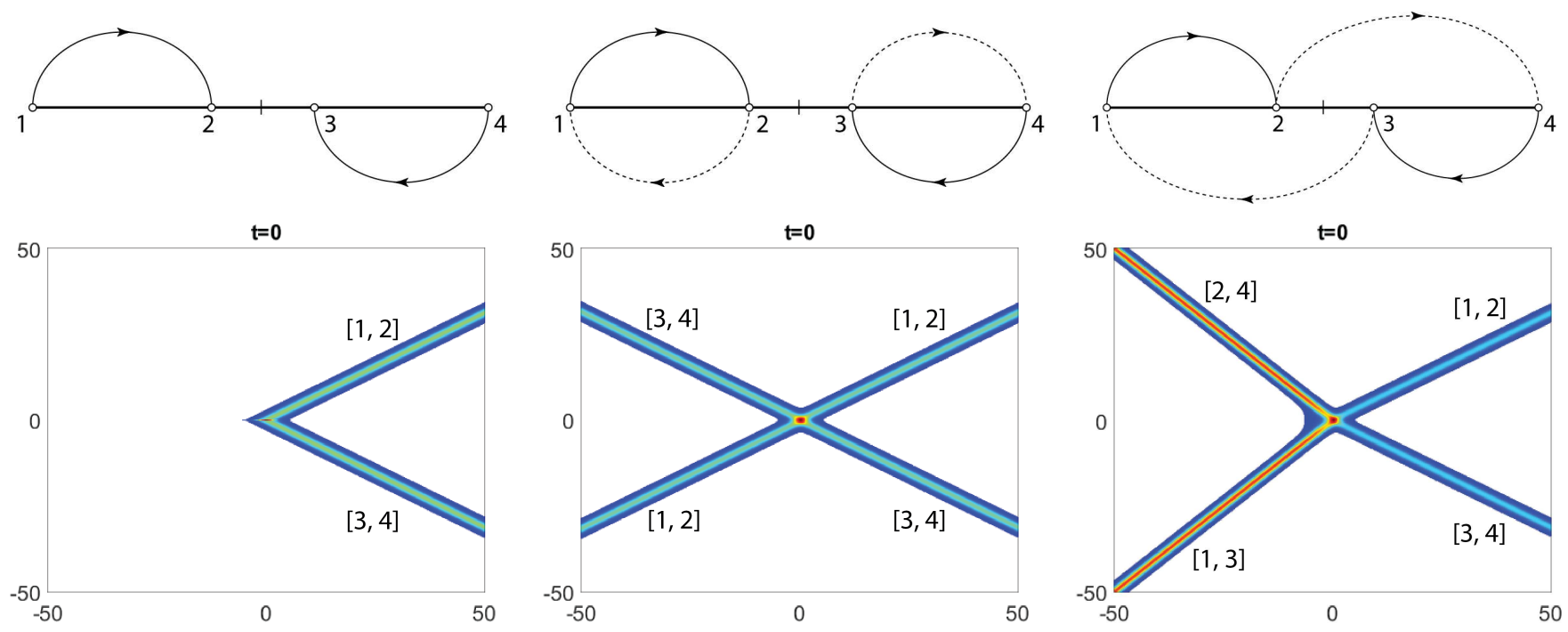

Figure 6: Possible chord completions of a partial chord diagram representing a $<$-shape initial data and the corresponding $(2,2)$-soliton solutions.

The completed chord diagram corresponds to a derangement $\pi$ representing the asymptotic solitons of the exact solution. However, the completion may not be unique as illustrated by the example in Figure 6 . The left frame on the top panel shows an partial chord configuration representing a $<$-shape initial condition consisting of a [1,2]-soliton for $y \gg 0$ and a [3,4]-soliton for $y \ll 0$ as shown on the left frame of the bottom panel. The partial chord configuration can be completed to form a derangement in two distinct ways as shown by the chord diagrams in the middle and right frames of Figure 6 . The completed chord diagrams correspond to a (2143)-type and a (2413)-type (2,2)-soliton as shown in the bottom panel. In [12], a minimal completion of the chords was first conjectured which states that a given initial condition evolves to the unique exact solution associated with the derangement $\pi$ whose chord diagram has the minimum total chord length. In Figure 6, the derangement with minimum chord length corresponds to $\pi=(2143)$ (middle frame). Then the conjecture predicts that the $<$-shape initial data in Figure 6 should evolve to a (2143)-type solution. This is also confirmed by numerical simulations [12, 13, 8].

Each of the initial conditions considered below evolves to an interacting wave pattern which converges to an exact $(2,2)$-soliton solution of KP. We clarify below precisely what we mean by convergence. First, we assume that the KP theory described in Section 2 holds for the evolving wave pattern. Hence, measuring the amplitudes $\frac{1}{2}\left(k_{j}-k_{i}\right)^{2}$ and slopes $k_{i}+k_{j}$ of the asymptotic solitons in this wave pattern allows us to identify each asymptotic soliton for $y \gg 0$ and $y \ll 0$. Using this information, we construct the derangement $\pi$ as explained in Section 2 and thus complete the partial chord diagram associated with the initial wave pattern. In all of our numerical simulations, we find that the full chord diagram is obtained by the minimal completion of the partial chord diagram. Once a particular type of a $(2,2)$-soliton solution is identified from the chord diagram, it is then possible to construct the corresponding exact solution from equations (3), (9) and the $A$-matrix given in Table 1 . Note however, that Table 1 gives only the form of the $A$-matrix in its reduced row-echelon form, but not the exact value of its entries. In order to find the numerical values of the $A$-matrix elements we proceed as follows: We define a local error function

$$
E(t)=\left[\frac{\iint_{B}\left|u_{\text {num }}(x, y, t)-u_{\text {exact }}(x, y, t)\right|^{2} d x d y}{\iint_{B} u_{\text {exact }}^{2}(x, y, t) d x d y}\right]^{\frac{1}{2}} .
$$

The $L^{2}$ norms are computed over a rectangular box

$$
B:=\left\{(x, y) \in \mathbb{R}^{2}:\left|x-x_{0}(t)\right| \leq l_{x},\left|y-y_{0}(t)\right| \leq l_{y}\right\},
$$


where $\left(x_{0}(t), y_{0}(t)\right)$ is normally chosen to be an intersection point of two (or more) interacting line solitons. The dimensions of the box $l_{x}, l_{y}$ are much smaller than the numerical domain; they are chosen such that the moving rectangle $B$ encloses the interaction region in the $x y$-plane for all $t>0$ during the simulation, and at the same time avoiding the effects from the boundary. The time-dependence of the interaction vertex $\left(x_{0}(t), y_{0}(t)\right)$ is determined from the explicit form of the KP soliton solution. For instance, in the Y-soliton example in Section 2, the point where all three line solitons intersect is taken to be the interaction vertex. We then construct the $A$-matrix by choosing its undetermined entries such that the error function $E(t)$ is minimized at a fixed time $t=T_{0}$. Note that this yields an exact KP soliton which is closest to the numerically computed wave form on the rectangle $B$ at $t=T_{0}$ in a local $L^{2}$-sense. We then confirm that the error, $E(t)$, decreases for $t>T_{0}$ with the same exact solution (i.e., whose $A$-matrix entries were optimized at $t=T_{0}$ ) up to sufficiently large time before the moving box $B$ gets too close to the boundary of the numerical domain.

In the following subsections, we present the results of our numerical simulations demonstrating the evolution of various types of solitary wave initial data to an exact solution of the KP equation. A typical initial data is formed by gluing together pieces of $[i, j]$ one-soliton solutions given by (5) for different values of the parameters $k_{i}$ and $k_{j}$. As mentioned earlier in the Introduction, numerical studies of the KP solitons were conducted using $<$ (sideways V)-shape initial data in previous works $[12,13,8]$ but they were not sufficiently exhaustive to cover all seven types of $(2,2)$-soliton solutions. Our work compliments the earlier work, and completes the numerical study of all $(2,2)$-soliton solutions of the KP equation.

Before presenting our new results, we would first like to discuss the numerical simulation of the Ysoliton which appeared in [8] (see also [13]) as an illustrative example. We believe this simple case will be useful to better understand the new simulations presented in the later subsections. Furthermore, we also use this example to show that the completion of partial chord diagrams is not a sufficient criterion to guarantee convergence. The proper choice of initial data that evolve to a stable KP soliton depends on other considerations which will be discussed using the Y-soliton example. This latter part also contains new numerical results for the Y-soliton, which does not appear in previous works.

\subsection{Y-soliton}

The Y-soliton solution was described earlier as an example in Section 2. It represents a resonant interaction of 3 one-soliton solutions. In our numerical experiments, we take the initial data consisting of a large amplitude [1,3]- and a small amplitude [1,2]-soliton glued together at the origin, as shown on the left frame of the top panel in Figure 7. Specifically, the initial condition is

$$
u(x, y, 0)=\left\{\begin{array}{cc}
0.18 \operatorname{sech}^{2} 0.3(x-y), & y>0 \\
1.28 \operatorname{sech}^{2} 0.8 x, & y \leq 0 .
\end{array}\right.
$$

with $\left\{k_{1}, k_{2}, k_{3}\right\}=\{-0.8,-0.2,0.8\}$. The middle frame (top panel) of Figure 7 shows the formation of a $[2,3]$-soliton as well as dispersive waves created due to the non-solitonic interaction of the initial waves at the intersection point. Note from Section 2 (the one-soliton case) that since $c_{i j}=\left(k_{i}^{2}+k_{i} k_{j}+k_{j}^{2}\right)>0$, the $x$-component of the velocity $V_{i j}$ of the $[i, j]$-soliton is always positive. That is, a line soliton propagates in the positive $x$-direction. On the other hand, the dispersive waves propagate in the negative $x$-direction. This can be seen from the dispersion relation of the KP equation (1) for a linear wave $\phi=\exp (i \mathbf{k} \cdot \mathbf{x}-i \omega t)$ with wave-vector $\mathbf{k}=\left(k_{x}, k_{y}\right)$ and frequency $\omega=\left(3 k_{y}^{2}-k_{x}^{4}\right) / 4 k_{x}$. The group velocity of the dispersive wave is then given by

$$
\mathbf{v}=\left(\frac{\partial \omega}{\partial k_{x}}, \frac{\partial \omega}{\partial k_{y}}\right)=\left(-\frac{3}{4}\left(k_{x}^{2}+\frac{k_{y}^{2}}{k_{x}^{2}}\right), \frac{3}{2} \frac{k_{y}}{k_{x}}\right) .
$$



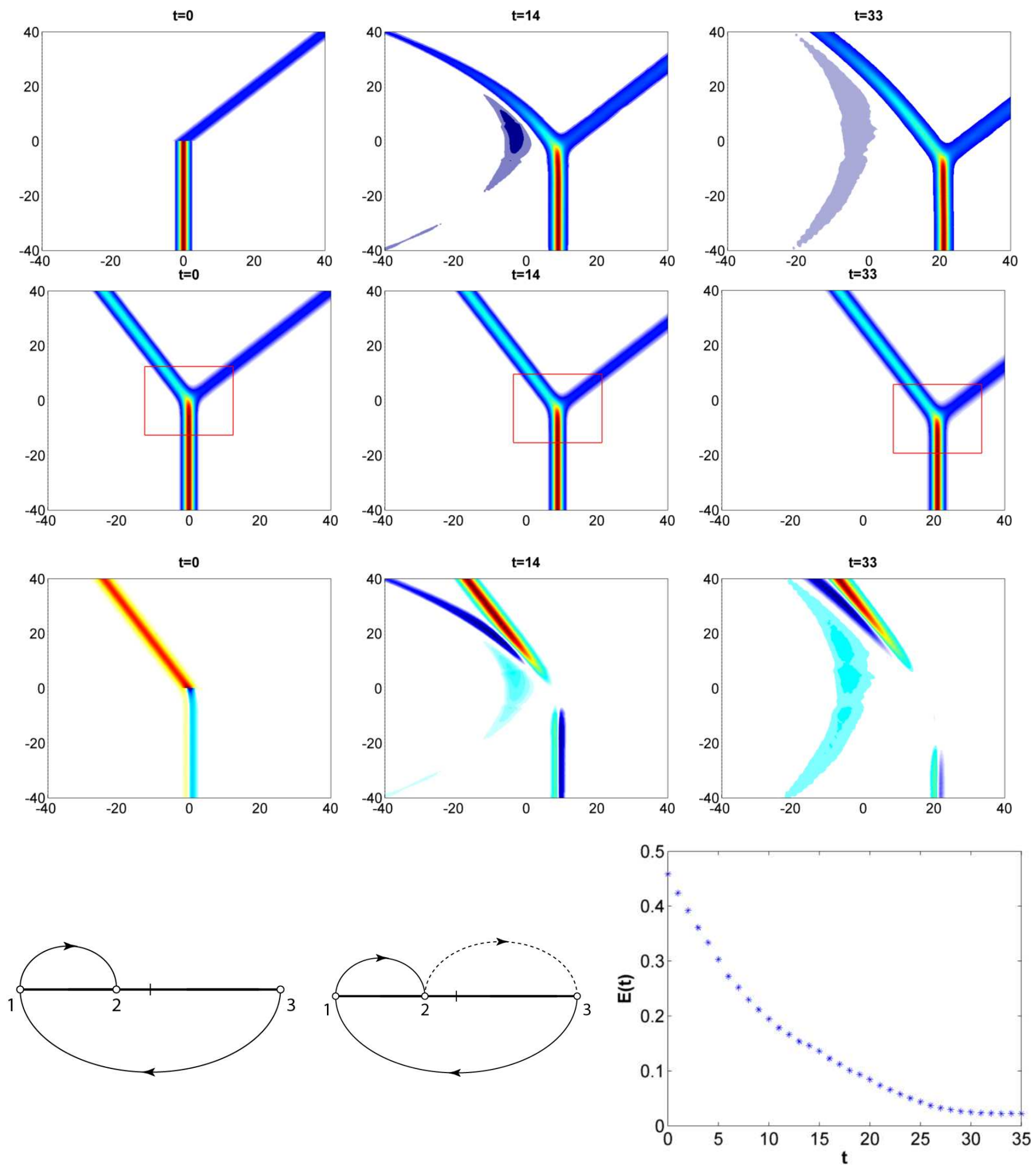

Figure 7: Simulation of a Y-soliton. Numerical domain $[-192,192] \times[-78,78]$. Top panel: numerical simulation of the solution $u_{\text {num. }}$. Second panel: evolution of the exact solution $u_{\text {exact }}$ with optimized parameters. Third panel: evolution of the function $v=u_{\text {exact }}-u_{\text {num. }}$. Bottom panel: Incomplete chord diagram and its completion. Error $E(t)$ vs $t$ minimized at $t=27$ (right frame).

Hence, the radiation disperses away from the near field region where the soliton interactions take place, asymtotically in time. In our numerical simulations, we observe that any finite neighborhood of the ver- 
tex of the numerical Y-soliton eventually separates from the dispersive waves as seen from the top panel of Figure 7. The first two figures in the bottom panel of Figure 7 feature the incomplete chord diagram corresponding to the initial data and the complete chord diagram corresponding to the Y-soliton. The completion of the chord diagram predicts the formation of a $[2,3]$-soliton with a positive slope and amplitude larger than the $[1,2]$-soliton, which is confirmed by our numerical simulations. By measuring the amplitude $\frac{1}{2}\left(k_{j}-k_{i}\right)^{2}$ and the slope $k_{i}+k_{j}$ of the asymptotic solitons in the numerical solution, we find that the $k$-parameters are almost exactly the same (the maximum error is less than $10^{-4}$ ) as the initial values $\left\{k_{1}, k_{2}, k_{3}\right\}=\{-0.8,-0.2,0.8\}$.

Next we demonstrate the convergence of our numerical solution to an exact Y-soliton following the procedure described in Section 3.1. The exact Y-soliton $\tau$-function is given by (6). We optimize the parameters $a$ and $b$ in the $A$-matrix so that the local $\mathrm{L}^{2}$ error defined in (10) is minimized. It is more convenient instead to vary the soliton parameters $x_{13}$ and $x_{12}$ which are related to the parameters $(a, b)$ via the formulas given in Section 2.2. Since a small change in $x_{i j}$ causes an exponentially large change in $(a, b)$, a higher accuracy in the optimization process can be achieved by varying the $x_{i j}$. We take a rectangular box $B$ with $l_{x}=21$ and $l_{y}=27.5$ as shown in the second panel of Figure 7 . The error is minimized at $\mathrm{t}=15$ to yield the optimized values of the parameters $\left(x_{12}, x_{13}\right)=(0.036,-0.138)$ which corresponds to the $A$-matrix

$$
A=\left(\begin{array}{ccc}
1 & 0 & -0.748 \\
0 & 1 & 0.478
\end{array}\right) .
$$

As explained in Section 3, this minimization scheme identifies an exact Y-soliton solution with the above $k$-values and $A$-matrix that is closest to the numerical solution in a local $\mathrm{L}^{2}$ sense. However, note that the line solitons in this exact solution have position shifts given by the non-zero optimized values of $x_{12}$ and $x_{13}$ above, whereas $x_{12}=x_{13}=0$ for the initial data. The second panel in Figure 7 shows the evolution of the constructed exact solution with the optimized parameters and the rectangular box $B$ of size $25 \times 25$ enclosing the interaction region. The center of the box $B$, taken as the common intersection point of the three resonantly interacting line solitons, is given by $x_{0}(t)=0.64 t-0.138$ and $y_{0}(t)=-0.2 t-0.174$ using (8). During the formation of the numerical Y-soliton, each of its asymptotic solitons experience a local shift which travel along its wave crest. As these shifts escape from the interaction region $B$, the numerical wave form converges to an exact Y-soliton solution with position shifts $x_{i j} \neq 0$. This fact is confirmed as we compute the error, $E(t)$, for larger times until $t=35$ and observe that the error decreases monotonically as shown in the bottom panel of Figure 7.

The optimized values $\left(x_{12}, x_{13}\right)=(0.036,-0.138)$ imply that the $[1,3]$-soliton suffers a negative position shift due to its interaction with the dispersive waves and loss of momentum due to the generation of the [2,3]-soliton at $x<0$. The [1,2]-soliton, on the other hand, has a small but positive shift as it gains energy from the high amplitude [1,3]-soliton. Then from (7), one finds that $x_{23}=-0.242$ as the shift for the generated $[2,3]$-soliton.

The generation of a $[2,3]$-soliton and dispersive waves leading to the convergence of the initial data to a Y-soliton inside the interaction region $B$ in the above numerical simulation may be explained by a heuristic argument given in [13]. If one decomposes the exact solution as $u_{\text {exact }}=u+v$ where $u$ solves (1) with the $[2,3]$ - and $[1,3]$-solitons as initial waves, then $v$ satisfies

$$
\left(4 v_{t}+6 v v_{x}+v_{x x x}\right)_{x}=-6(u v)_{x x}
$$

which is a "forced" KP equation. The product $u v$ in the right hand side forcing term has only support near the origin at $t=0$ if one assumes that the initial solitons in $u$ evolve exactly as their counterparts in $u_{\text {exact }}$. Under this assumption, one expects $v$ to evolve according to the KP equation (1) with initial condition which contains a [2,3]-half-soliton for $y>0$ and $x<0$. Since the lower half (for $x>0$ ) of the [2,3]-soliton is missing, the front edge of the upeer half experiences a strong dispersive effect. Consequently, this edge 

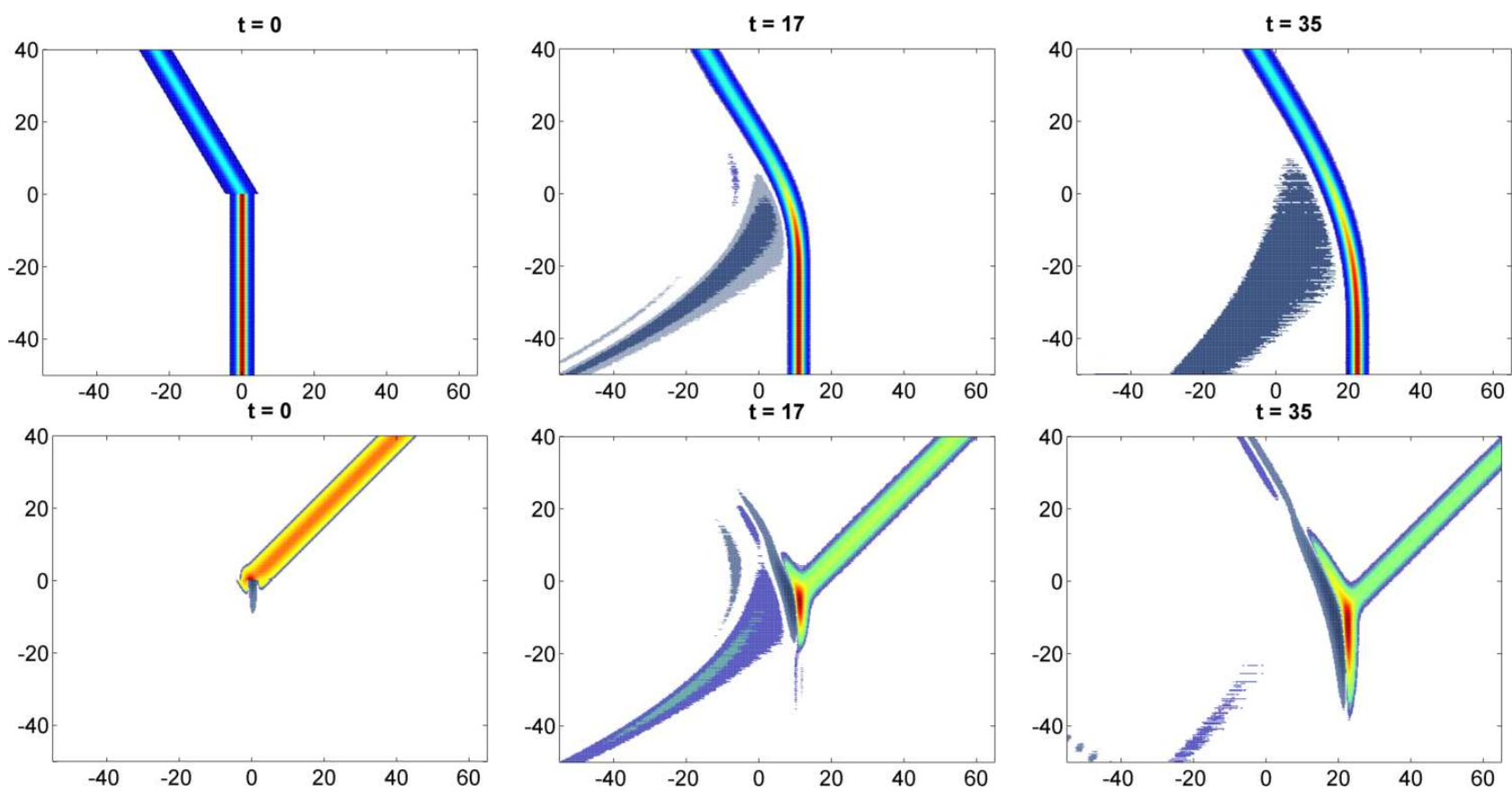

Figure 8: Evolution of initial condition not converging to a Y-soliton. Top panel: formation of a virtual $[1,2]$-soliton for $y<0$. Bottom panel: Evolution of the function $v=u_{\text {exact }} u_{\text {num. }}$. See text for details.

decays emitting dispersive waves which propagate in the negative $x$-direction. Then the decay of the [2,3]half-soliton in the solution $v$ implies the emergence of this soliton in the solution $u=u_{\text {exact }}-v$. The third panel in Figure 7 shows the evolution of the solution $v=u_{\text {exact }}-u$ where the exact solution is the Y-soliton with the optimized values of $x_{i j}$ obtained above and $u$ is the numerical solution. The initial condition for $v$ (left frame) consists of the [2,3]-soliton for $y>0$ but also a waveform at the [1,3]-soliton location for $y<0$. This waveform is there because the $[1,3]$-soliton in the exact solution $u_{\text {exact }}$ has a negative position shift $\left(x_{13}=-0.138\right)$ but $x_{13}=0$ in the initial condition for $u$. The next two frames show the decay of the solution $v$ into dispersive radiation. Note in particular that the neighborhood of the interaction vertex, where $v \rightarrow 0$ i.e., $u$ converges to $u_{\text {exact }}$, is increasing asymptotically in $t$. We emphasize that the argument given above is not rigorous, we plan to provide a more mathematically exact argument along these lines in the future.

Non-convergence results: The above numerical simulation described the evolution of a particular initial condition to a Y-soliton as predicted by the unique completion of the corresponding partial chord diagram. However, chord completion alone is not sufficient to guarantee the convergence of any initial data to a KP soliton. That is, there exist initial conditions which do not converge a KP soliton even if the completion of their associated partial chord diagrams predict so, as we describe via the examples below.

Case 1. Let us consider an initial condition shown on the left frame of the top panel in Figure 8 where a $[2,3]$-soliton for $y>0$ is glued together at the origin with a $[1,3]$-soliton for $y<0$, with the same $k$-parameter values as above. The partial chord diagram is similar as in Figure 7 except that the up-chord is 23 instead of 12 . Then from the unique completion of this diagram one would expect that a [1,2]soliton with a negative slope to form for $y>0$ if this initial condition were to evolve to a exact Y-soliton. However, in order for it to form for $x>0$, the $[1,2]$-soliton has to be created as a small perturbation from the intersection vertex ahead of the initial solitons. But this is impossible since any small perturbation tend to disperse toward the negative $x$-direction with group velocity $\mathbf{v}$ as mentioned above. This is also confirmed by our simulation in Figure 8 which shows that the $[1,2]$-soliton is not formed for $t>0$. Instead, the intersection point of the two initial solitons smooth out after shedding dispersive waves and forms a 
bend at the point where the [1,2]-soliton would have otherwise formed for $x>0$.

The $[1,2]$-soliton which is not formed in this case can be treated as a virtual soliton which is not visible. This notion can be understood also using the decomposition $u_{\text {exact }}=u+v$ discussed in the context of the earlier simulation of the Y-soliton. The bottom panel of Figure 8 shows the evolution of $v=u-u_{\text {exact }}$ where $u$ is the numerical solution and $u_{\text {exact }}$ in this case is a Y-soliton with $x_{i j}=0$. The simulation shows that $v$ evolves as a $[1,2]$-soliton for $x>0$ while its edge extends to the lower-half plane for $x<0$ and decays for $t>0$ with a bow-shape wake dispersing toward the negative $x$-axis. This implies that the $[1,2]$-soliton appears for $x>0$ in both $u_{\text {exact }}$ and $v$, hence it can not appear in the solution $u=u_{\text {exact }}-v$ for $x>0$ as it cancels out. Furthermore, a virtual (negative wave) [1,2]-soliton appears for $x<0$ in the solution $u$ as seen from its evolution in the top panel of Figure 8. Note that the assumption $v=u-u_{\text {exact }}$ represents the $[1,2]$-soliton for $x>0$ holds at $t=0$ but is not entirely correct for $t>0$ because the shifts $x_{i j}$ experienced by the initial solitons in $u$ during the time evolution can be large while $x_{i j}=0$ for the exact solution $u_{\text {exact }}$. This discrepancy is shown in in the simulation as well where in fact $v=u-u_{\text {exact }}$ evolves to a Y-shape near the interaction vertex implying that the wave-crests of the [1,3]- and [2,3]-soliton in $u$ experience a positive shift.
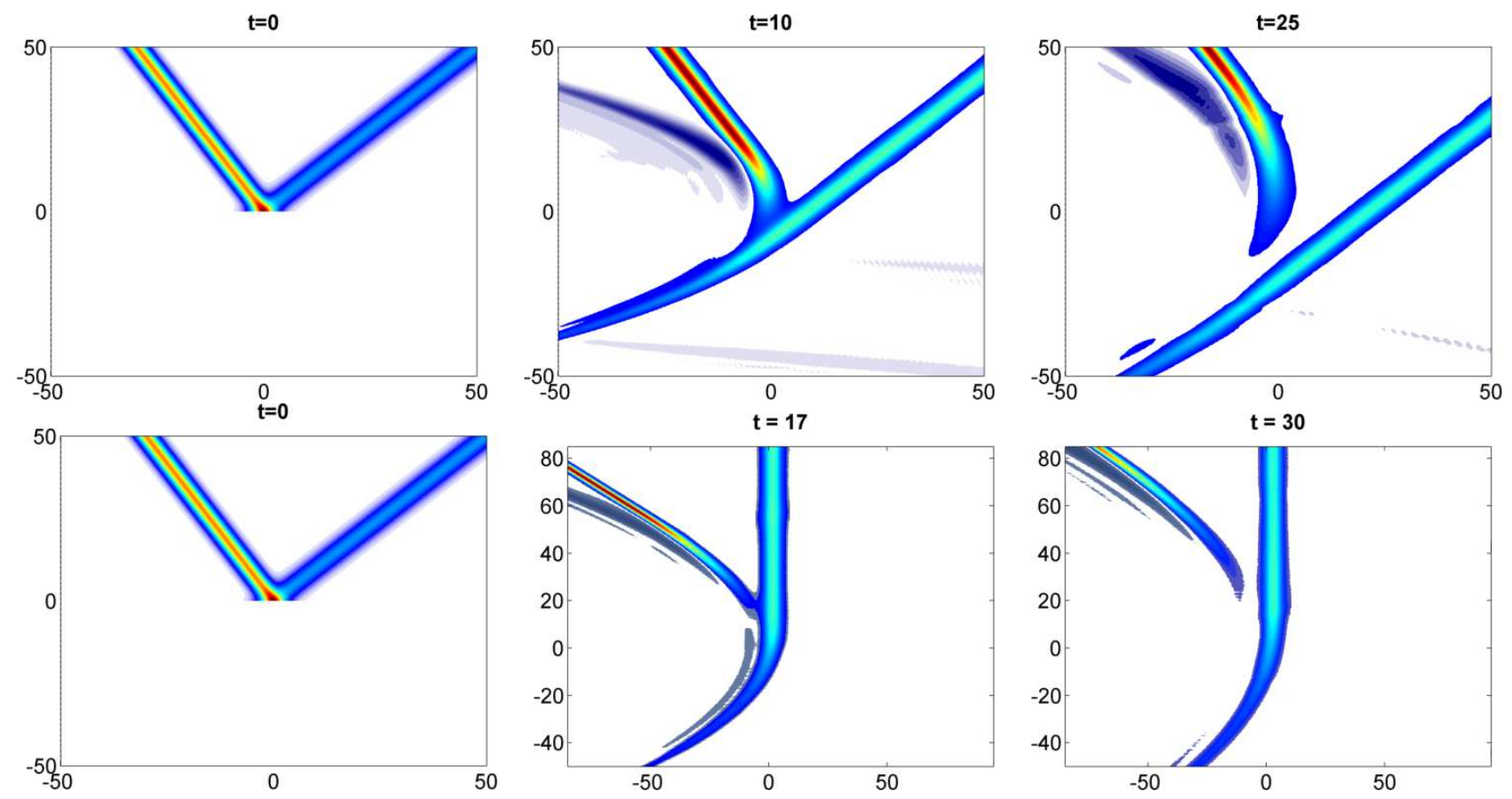

Figure 9: Non-convergence result showing the separation of the initial [1,2]- and [2,3]-soliton at the intersection point.

Case 2. Here we take the $[1,2]$ - and $[2,3]$-solitons for $y>0$ with with the same $k$-parameters, i.e., $\left\{k_{1}, k_{2}, k_{3}\right\}=\{-0.8,-0.2,0.8\}$ as our initial condition which corresponds to a partial chord diagram with two up-chords and the $\overparen{13}$ down-chord missing. Again, the unique chord completion implies the formation of a $[1,3]$-soliton for $y<0$ due to a three-wave interaction at the intersection point. But our numerical simulation in Figure 9 (top panel) shows that a [1,3]-soliton is not generated in this case. Here, the [1,2]soliton travels (in a direction normal to its wave-crest) to the right and in the negative $y$-direction with a speed $c_{12}=0.84$; whereas the $[2,3]$-soliton travels to the right but in the positive $y$-direction with speed $c_{23}=0.52$. Consequently, they separate from each other quickly at the intersection point and as a result, fails to generate a $[1,3]$-soliton by resonant interaction. As the solitons separate, the [2,3]-half-soliton decays with its edge getting shorter and radiating dispersive waves, while the $[1,2]$-soliton grows. We have 
also considered other initial conditions where the $[2,3]$-soliton travels faster than the $[1,2]$-soliton. The bottom panel of Figure 9 displays such a situation with $k$-values $\left\{k_{1}, k_{2}, k_{3}\right\}=\{-0.3,0.3,1.3\}$. But the $[2,3]$-soliton still detaches from the intersection point because it always moves in the positive $y$-direction due to its positive slope.

The above examples demonstrate that the unique completion of a partial chord configuration is a necessary but not sufficient condition that guides the choice of initial data which converge to a KP soliton. This condition needs to be supplemented by additional constraints as evident from these examples. In choosing the initial conditions, one needs to observe that (a) it is impossible to form a soliton ahead of the initial soliton wave-front via nonlinear interactions, and (b) the soliton velocities should be adjusted such that the initial solitons do not separate from their intersection point. These considerations, although perhaps not exhaustive, play important roles in our choice of initial data for the new numerical simulations presented below.

\section{3 (4312)-type soliton}

In this example, we consider initial data which is obtained by gluing three semi-infinite line solitons at the origin (see left frame in the top panel of Figure 10 below). Specifically, we choose

$$
u(x, y, 0)= \begin{cases}2 \operatorname{sech}^{2} x, & y \geq 0, \\ 1.62 \operatorname{sech}^{2} 0.9(x-0.2 y)+1.25 \operatorname{sech}^{2} 0.75(x+0.5 y), & y<0 .\end{cases}
$$

This corresponds to a [1,4]-half-soliton in the upper-half of the $x y$-plane, which is glued together at the origin with [1,3]-half-soliton $(x<0)$ and a $[2,4]$-half-soliton $(x>0)$ in the lower-half of the $x y$-plane. The four $k$-parameters are chosen to be $\left\{k_{1}, k_{2}, k_{3}, k_{4}\right\}=\{-1,-0.5,0.8,1\}$. The bottom panel of Figure 10 shows the incomplete chord diagram (left frame) corresponding to the above initial condition. Our numerical simulations then demonstrate the formation of a (4312)-type soliton which is also illustrated by the completion of chords in the middle frame of the bottom panel of Figure 10. Note that this chord diagram is completed in a unique fashion by connecting the up-chord (23).

The top panel in Figure 10 shows the evolution of the initial data. The large amplitude [1,4]-soliton and the $[2,4]$-soliton in $x \geq 0$ (right half plane) interact resonantly to create an intermediate $[1,2]$-soliton in the lower-half $x y$-plane. Note that the initial [1,3]- and [1,4]-soliton resonant interaction is forbidden as it would create a $[3,4]$-soliton for $y<0$ ahead of the initial waves since $k_{3}+k_{4}>0$. This type of non-convergence result was discussed for the Y-soliton case in Section 3.2. Here, the $[1,4]-,[2,4]$ - and the $[1,2]$-solitons form a $X$-shape. Since the slope of the $[1,2]$-soliton is negative $\left(\tan \Psi_{12}=k_{1}+k_{2}=-1.5\right)$ it is created behind the $[1,4]$ - and $[2,4]$ wavefronts. Then the $[1,2]$-soliton interacts resonantly with the $[1,3]$-soliton in the lower-half $x y$-plane to form the asymptotic $[2,3]$-soliton for $y>0$. Note that we chose $k_{2}+k_{3}>0$ so that the $[2,3]$-soliton is formed behind the $[1,4]$-soliton. The final wave patterns shown in the right frame of the top panel of Figure 10 consist of a Y-soliton and a $X$-soliton bridged by a common intermediate $[1,2]$-soliton. The middle frame shows the formation of the intermediate $[1,2]$-soliton and the asymptotic $[2,3]$-soliton together with dispersive waves which are created due to the non-solitonic interaction of the initial waves at the intersection point. As in the Y-soliton case in Section 3.2, the numerically generated (4312)-type soliton wave-form moves to the right and eventually separates from the dispersive waves as seen from the right frame of the top panel of Figure 10. We also observe that the initial $[1,4]$-soliton experiences a shift of its center in the positive $x$-direction due to its interaction with the dispersive radiation, and this shift travels along the $[1,4]$-soliton along the positive $y$-axis. We confirm the formation of a (4312)-type soliton numerically by measuring the amplitudes $\frac{1}{2}\left(k_{j}-k_{i}\right)^{2}$ and the slopes $k_{i}+k_{j}$ of the asymptotic solitons of the wave pattern in the numerical solution. 

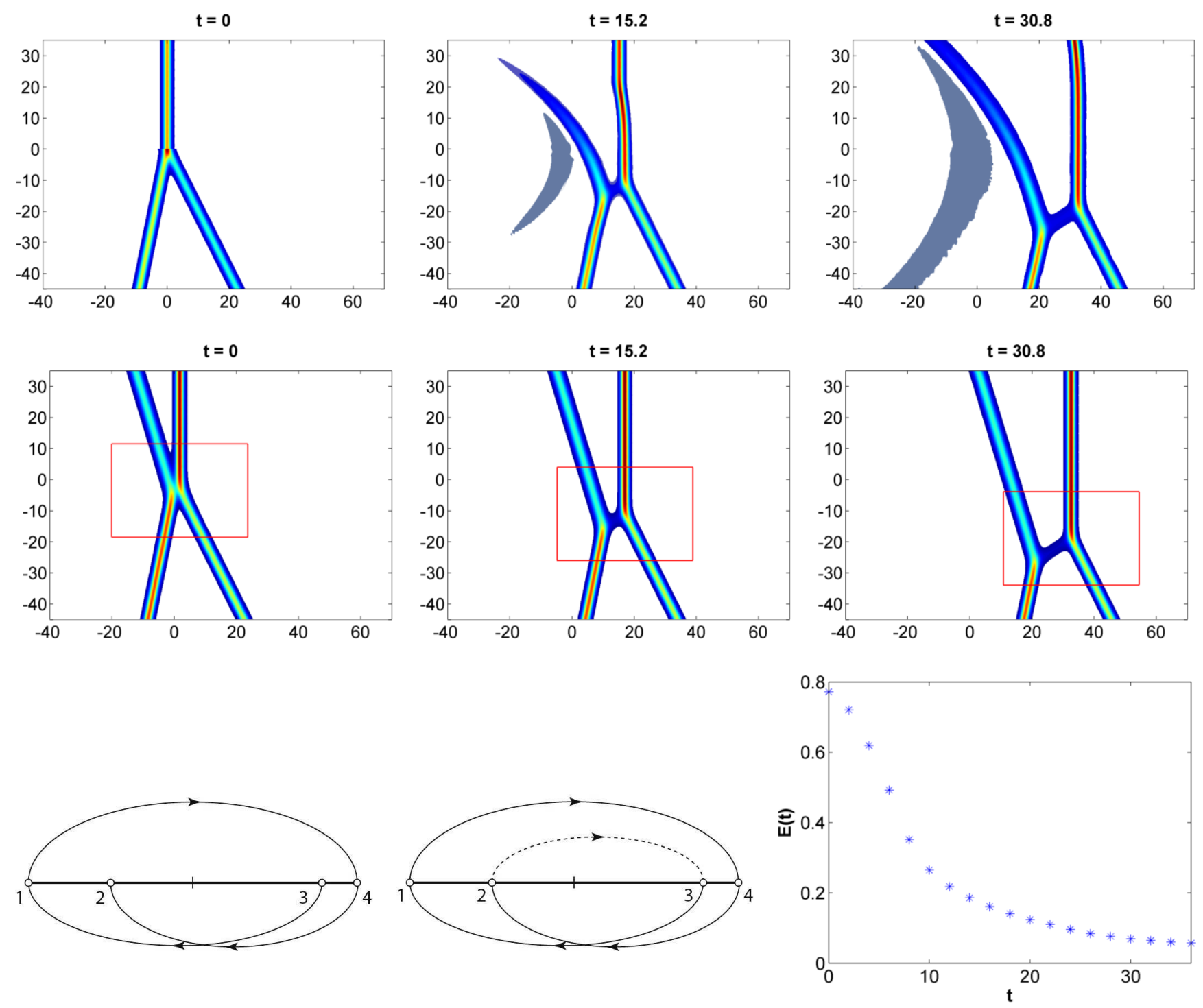

Figure 10: Simulation of a (4312)-type soliton. Domain $[-192,192] \times[-78,78]$. Top panel: Numerical evolution of initial data. Middle panel: Evolution of an exact (4312)-soliton with optimized parameters. Bottom panel: (4312)-Chord diagrams with chord $\overparen{23}$ missing from the initial diagram, and error curve, $E(t)$ minimized at $t=27$.

Next, we demonstrate the convergence of our numerical solution to an exact (4312)-type soliton. We construct the exact (4312)-type solution from its $\tau$-function in (9), the $k$-parameters, and the $A$-matrix. One finds from Table 1 (Appendix A) that the (4312) $A$-matrix has three positive parameters $a, b$, and $c$ related to the soliton parameters ( $x$-intercepts) $x_{i j}$ of the asymptotic solitons $[1,4],[1,3]$, and $[2,4]$. We optimize these parameters by minimizing the error function, $E(t)$, at $t=27$ to obtain the optimized values $x_{13}=0.508, x_{14}=1.726, x_{24}=-0.016$ which yield the following $A$-matrix for the exact (4312)-type solution

$$
A=\left(\begin{array}{cccc}
1 & 0 & -0.154 & -0.285 \\
0 & 1 & 3.599 & 0
\end{array}\right)
$$

The middle panel in Figure 10 shows the evolution of the exact solution with this $A$-matrix and parameters $\left\{k_{1}, k_{2}, k_{3}, k_{4}\right\}=\{-1,-0.5,0.8,1\}$. The box size for this numerical simulation is $43.75 \times 30$, and the box is centered at the $X$-vertex $\left(x_{0}(t), y_{0}(t)\right)$ formed by the $[1,2]-,[1,4]$ - and the $[2,4]$-solitons with $x_{0}(t)=$ $t+1.726$ and $y_{0}(t)=-0.5 t-3.48$ (see (8)). The right frame of the bottom panel in Figure 10 shows the error $E(t)$ which decreases monotonically in time. The line solitons in this exact solution have non-zero 
position shifts given by the optimized values of $x_{14}, x_{13}$ and $x_{24}$ above. These non-zero position shifts are observed from the numerical simulations as well. Note that the $[1,4]$-soliton is pushed forward, as seen from the top panel (middle frame) of Figure 10, due to the nonlinear interaction. This accounts for its large positive shift $x_{14}=1.726$. The positive shift of the $[1,3]$-soliton is due to its resonant interaction with the $[1,2]$ - and the [2,3]-soliton. The [2,4]-soliton loses momentum due to the formation of the $[1,2]$-soliton for $x<0$ and suffers a small shift in the negative $x$-direction. Thus, each of the asymptotic solitons of the (4312)-type solution experiences a position shift $x_{i j} \neq 0$ similar to the Y-soliton in the previous subsection. We remark here that if we choose an initial data obtained by reflecting the initial data in this example about
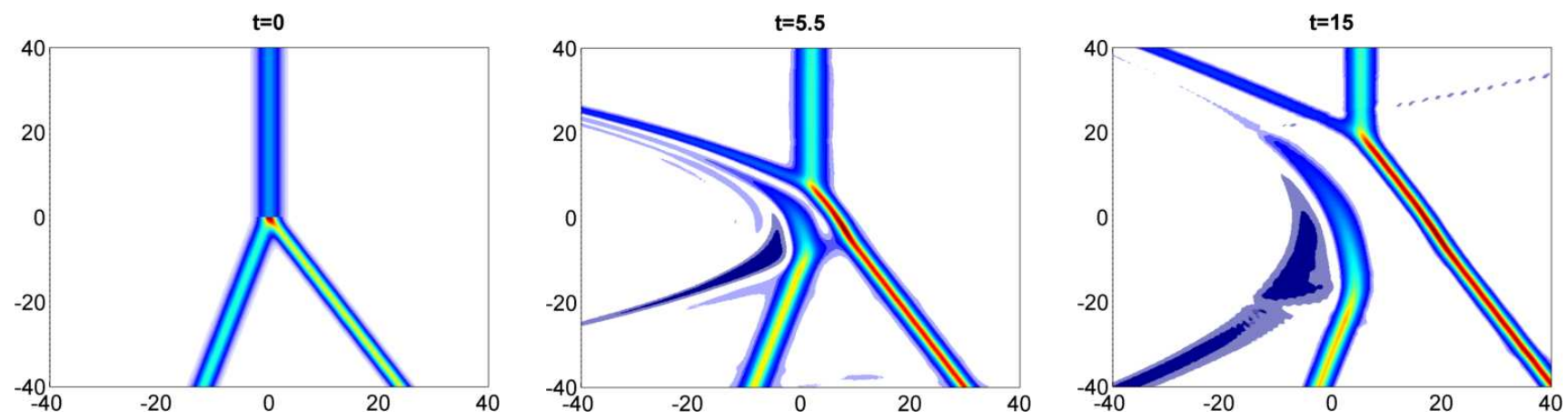

Figure 11: Simulation of an (4312)-type soliton. Here, the initial condition consists of a [2,3]-soliton instead of $[1,4]$-soliton for $y>0$ as in the previous example.

the $x$-axis, then our numerical simulation shows the formation of a (3421)-type KP soliton solution. The (3421)-type solution is dual to the (4312)-type solution as explained in Appendix A. We do not include the (3421)-type example since it is similar to the present case.

In our next simulation, we try to create a (4312)-type soliton from an initial condition obtained by replacing the initial $[1,4]$-soliton by a $[2,3]$-soliton for $y>0$. We choose the $k$-parameters in this case to be $\left\{k_{1}, k_{2}, k_{3}, k_{4}\right\}=\{-0.9,-0.6,0.6,1.2\}$. Specifically, the initial wave-form consists of a $[2,3]$-soliton with slope $k_{2}+k_{3}=0$ for $y>0$ joined together at the origin to a positive slope $[2,4]$-soliton and a negative slope $[1,3]$-soliton for $y<0$ as shown in Figure 11. The corresponding chord diagram is similar to in Figure 10 except that the up-chord $\overparen{14}$ is replaced by 23 . Then from the chord completion we expect that a high amplitude [1,4]-soliton should be created for $y>0$ with a positive slope since $k_{1}+k_{4}>0$. However, the evolution of the initial condition fails to generate a stable $[1,4]$-soliton in this case. This can be explained as follows by analyzing the underlying soliton interactions. First consider the resonant interaction between the [2,3]- and [1,3]-soliton forming a [1,2]-soliton. This is not possible because the $[1,2]$-soliton would have to be formed with a negative slope for $y>0$ in front of the $[2,3]$ - and $[1,3]$ solitons. Instead, the $[2,3]$-soliton interacts with the $[2,4]$-soliton resonantly to generate a $[3,4]$-soliton with a positive slope for $y>0$. Now this $[3,4]$-soliton need to resonantly interact with the $[1,3]$-soliton in the lower-half plane to generate the desired $[1,4]$-soliton for $y>0$. But this does not happen as the [3,4]soliton travels much faster $\left(c_{34}=2.52\right)$ than the $[1,3]$-soliton and the two solitons quickly separate from each other. Finally, the wave-form splits into two parts as the Y-soliton (formed by the [2,3]-, [2,4]-, and $[3,4]$-solitons) separate from the $[1,3]$-soliton which decays. These interactions can be seen from the plots in the middle and right frames of Figure 11. The middle frame also shows the temporary formation of a $[1,2]$-soliton due to the resonant interaction between the $[1,3]$ - and $[2,3]$-solitons. The interaction between the initial $[1,3]$ - and $[2,4]$-soliton can also create a [1,2]-soliton for instance, in the case of (3412)-type soliton (see third frame in Figure 5). But this [1,2]-soliton can not interact with the faster [2,4]-soliton to its right to form a $[1,4]$-soliton. Consequently, the $[1,2]$-soliton dissipates for larger $t$ values. This result is similar to the non-convergence results described in Section 3.2 and shows that not all initial conditions would converge to a (4312)-type soliton although it may seem possible from the perspective of completion 
of a partial chord diagram.

\section{4 (4321)-type soliton}

We consider another example of initial data consisting of three semi-infinite waves coincident at the origin. In this case the initial wave form is chosen as
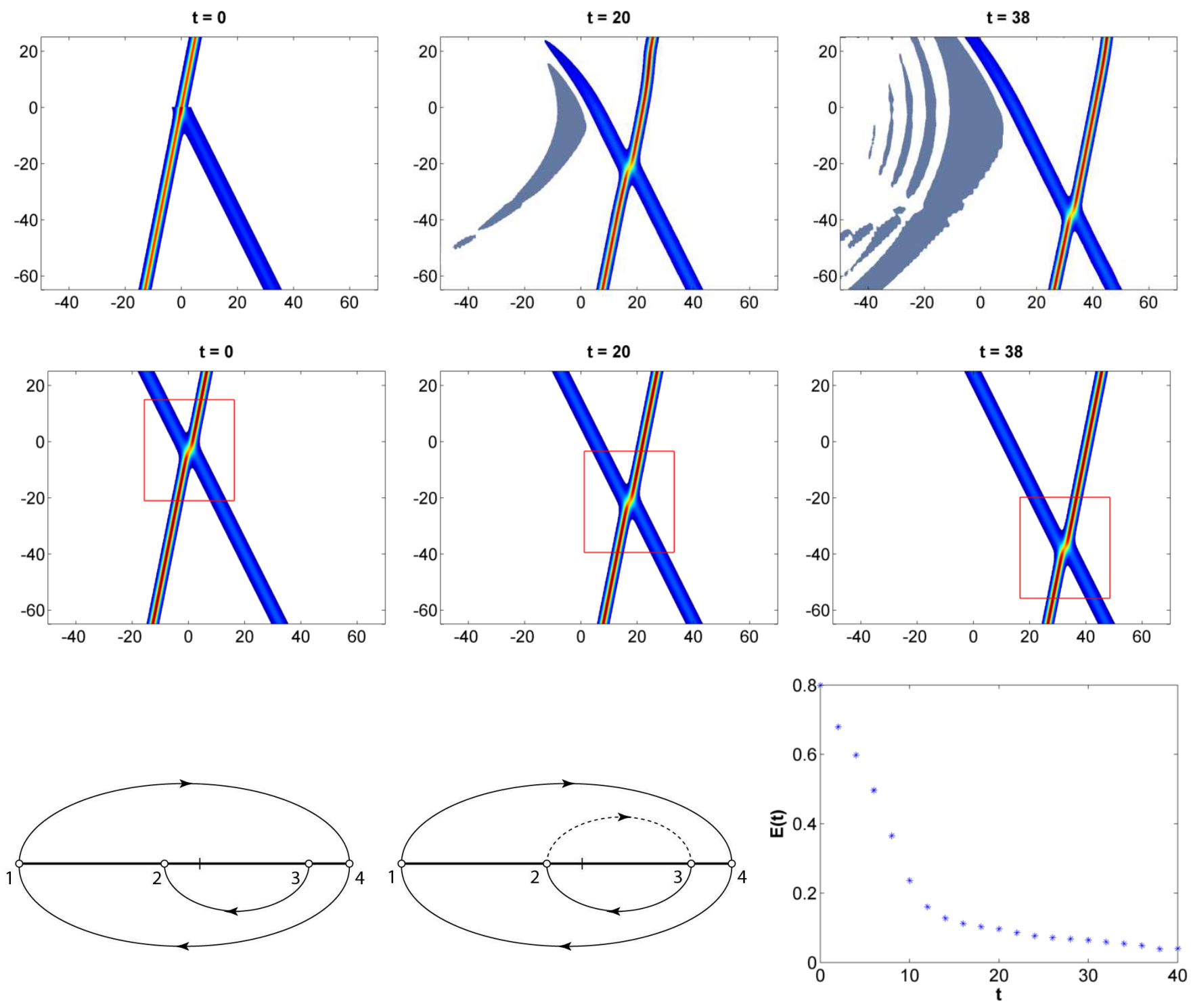

Figure 12: Simulation of an (4321)-type soliton. Top panel: Numerical evolution of initial data. Middle panel: Evolution of an exact (4321)-soliton with optimized parameters. Bottom panel: Chord diagrams and error curve, $E(t)$ minimized at $t=30$. Numerical domain: $[-192,192] \times[-150,150]$

$$
u(x, y, 0)= \begin{cases}2 \operatorname{sech}^{2}(x-0.2 y), & y \geq 0 \\ 0.405 \operatorname{sech}^{2} 0.45(x+0.5 y)+2 \operatorname{sech}^{2}(x-0.2 y), & y \leq 0\end{cases}
$$

which corresponds to an infinite $[1,4]$-soliton glued together at the origin with a semi-infinite $[2,3]$-soliton in the lower half plane. The initial condition is shown in the top panel (left frame) of Figure 12. The $k$ parameters chosen here are $\left\{k_{1}, k_{2}, k_{3}, k_{4}\right\}=\{-1.1,-0.2,0.7,0.9\}$ such that $\tan \Psi_{14}=-0.2$ and $\tan \Psi_{23}=$ 0.5 . That is, the $[1,4]$-soliton makes a negative angle with the $y$-axis, and it propagates towards $x>0$ and 
$y<0$. The simulation shows that the interaction between the solitons near the origin generates dispersive waves which move to the left in a direction opposite to the $[1,4]$-soliton. The $[1,4]$-soliton for $y>0$ is pushed forward and undergoes a position shift which travels along its top half, as shown in the middle frame of the top panel in Figure 12. In addition, a new [2,3]-half-soliton is formed behind the [1,4]soliton in the upper half-plane. This is confirmed by measuring the amplitude and slope of the newly generated solitary wave. As the dispersive waves separate further from the wave pattern the $[2,3]$-soliton is fully formed and the shift along the [1,4]-soliton disperses toward the boundary along the positive $y$ direction. The resulting wave form shown on the right frame (top panel) of Figure 12 is a (4321)-type soliton consisting of a pair of interacting $[1,4]$ - and [2,3]-solitons forming an X-shape. The intersection point of the $X$-shape moves to the right and in the negative $y$-direction since the $[1,4]$-soliton is faster than the $[2,3]$-soliton. Our observations are confirmed from the completion of the incomplete chord diagram as well as from the error function $E(t)$ shown on the bottom panel of Figure 12.

The error $E(t)$ is computed over a box of size $32 \times 36$ around the interaction region of the $[1,4]$ - and the $[2,3]$-solitons. The center of the box is located at the center of the interaction which turns out to be a local saddle point for $u(x, y, t)$. The location of the box center is given by $x_{0}(t)=0.85 t+0.26, y_{0}(t)=-0.91 t-$ 3.17. The error is minimized at $t=30$ which is a larger value of time since it takes a while for the [2,3]soliton for $x<0$ to fully form. We also needed to increase the domain size to $[-192,192] \times[-150,150]$ for this simulation (normal domain size $=[-192,192] \times[-78,78]$, see Appendix B). The curve for $E(t)$ in the bottom panel of Figure 12 demonstrates the monotonic decay of the error with time. The optimal values of the parameters that minimizes the error are given by $x_{14}^{+}=1.496$ and $x_{23}^{-}=0.009$ which are the $x$-intercepts at $t=0$ of the $[1,4]$-soliton in $y>0$ and $[2,3]$-soliton for $y<0$. The corresponding $A$-matrix calculated from Table 1 in Appendix $A$ is

$$
A=\left(\begin{array}{cccc}
1 & 0 & 0 & -0.452 \\
0 & 1 & 5.456 & 0
\end{array}\right) .
$$

The exact (4321)-type solution with this $A$ and the chosen $k$-values, i.e., $\left\{k_{1}, k_{2}, k_{3}, k_{4}\right\}=\{-1.1,-0.2,0.7,0.9\}$, are plotted in the middle panel of Figure 12.

From the numerical simulations we observe that the nonlinear interaction between the [1,4]- and [2,3]solitons are repulsive in nature in the sense that the two [1,4]-half-solitons for $x>0$ and for $x<0$ move away from each other, likewise for the two [2,3]-half-solitons as well. The asymptotic theory for KP gives an estimate of the net shift (see e.g. [7]) to be

$$
\Delta x_{14}:=x_{14}^{+}-x_{14}^{-}=-\frac{\ln \Delta_{P}}{k_{4}-k_{1}}, \quad \Delta x_{23}:=x_{23}^{-}-x_{23}^{+}=-\frac{\ln \Delta_{P}}{k_{3}-k_{2}}, \quad \Delta_{P}:=\frac{\left(k_{2}-k_{1}\right)\left(k_{4}-k_{3}\right)}{\left(k_{4}-k_{2}\right)\left(k_{3}-k_{1}\right)},
$$

where $x_{i j}^{ \pm}$are the shifts of the $[i, j]$-soliton for $y>0$ and $y<0$. The net shift is positive (repulsive) due to the ordering $k_{1}<k_{2}<k_{3}<k_{4}$ of the $k$-parameters for each of the [1,4]-, [2,3]-solitons. Furthermore, this shift depends only on the $k$-parameters but not on the $A$-matrix. In this example, $\Delta x_{14}=1.199, \Delta x_{23}=2.664$. Then the shifts of the [1,4]-, $[2,3]$-solitons for $y<0$ and $y>0$, respectively, are given by $x_{14}^{-}=x_{14}^{+}-\Delta x_{14}=$ 0.297 and $x_{23}^{+}=x_{23}^{-}-\Delta x_{23}=-2.655$. The formulas in (11) correspond to the net position shifts due to nonlinear interaction between the $[1,4]$ - and [2,3]-solitons. If the initial condition corresponded to an exact solution at $t=0$ with the interaction center at the origin, the position shifts of the individual solitons would be symmetric, i.e., $x_{14}^{+}=-x_{14}^{-}=\frac{1}{2} \Delta x_{14}=0.6$ and $x_{23}^{-}=-x_{23}^{+}=\frac{1}{2} \Delta x_{23}=1.332$. In contrast, the initial condition for our numerical simulation represents a strong perturbation (of the exact solution) at the intersection point at $t=0$. As a result, the $[1,4]$-soliton experiences a much larger shift in the positive $x$-direction while the $[2,3]$-soliton has an overall negative shift as it loses momentum due to the generation of its other half on the left of the $[1,4]$-half-soliton for $y>0$.

We also compute the amplitude at the center of the interaction region between the $[1,4]$ - and $[2,3]$ solitons and compare with the asymptotic value of the amplitude predicted by the KP theory. Figure 13 
shows that the amplitude at the interaction point decreases from the initial value of $A_{1}+A_{2}=2.405$, where $A_{1}=\frac{1}{2}\left(k_{4}-k_{1}\right)^{2}, A_{2}=\frac{1}{2}\left(k_{3}-k_{2}\right)^{2}$ are, respectively, the amplitudes of the $[1,4]-$ and $[2,3]$-solitons, to the theoretical value indicated by the horizontal line in the graph. The theoretical value of the amplitude at the interaction point is given by [7]

$$
u_{\text {int }}=A_{1}+A_{2}-2 \sqrt{A_{1} A_{2}}\left(\frac{1-\sqrt{\Delta_{P}}}{1+\sqrt{\Delta_{P}}}\right)
$$

where $\Delta_{P}$ was defined earlier in (11). From the $k$-parameter values used in this numerical experiment, we obtain $u_{\text {int }}=1.439$.

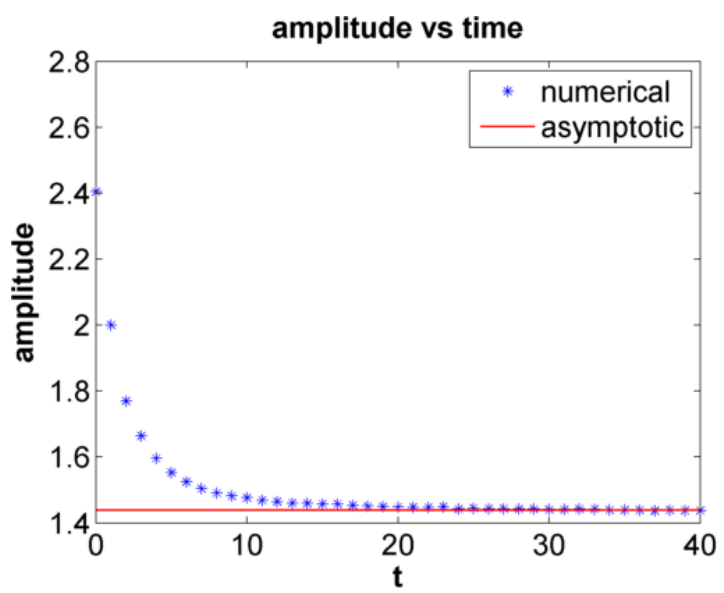

Figure 13: (4321)-soliton: Amplitude at the interaction center $u_{\text {int }}$ vs $t$.
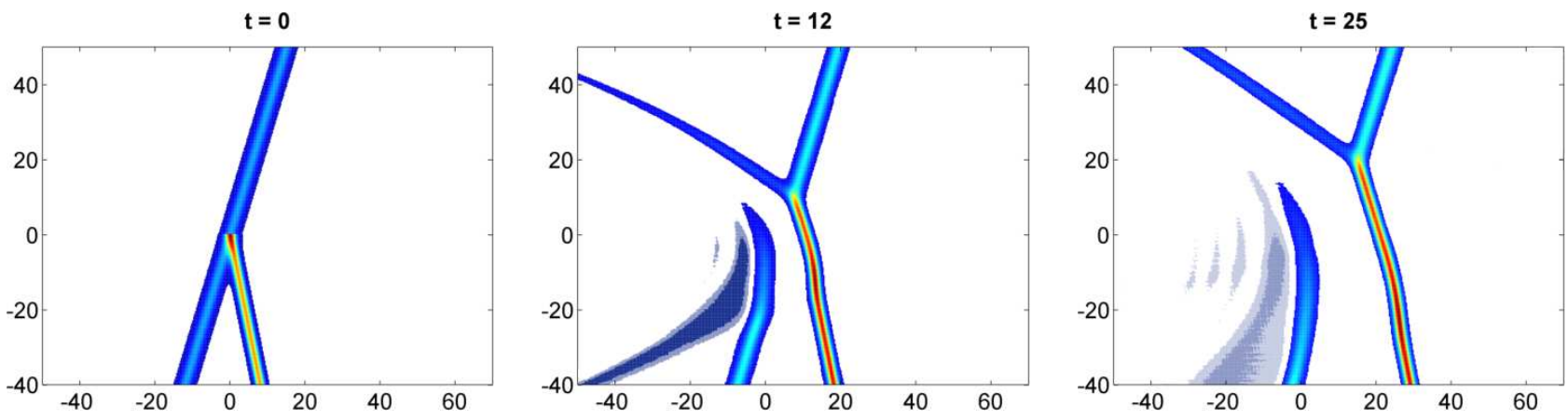

Figure 14: Non-convergence of initial data to a (4321)-type soliton.

Next, we consider initial conditions where the roles of the [1,4]- and [2,3]-solitons are switched in order to generate a (4321)-type soliton. That is, we consider a full [2,3]-soliton with a negative slope glued to a half $[1,4]$-soliton with a positive slope for $y<0$ as shown in the left frame of Figure. The $k$-values in this case are $\left\{k_{1}, k_{2}, k_{3}, k_{4}\right\}=\{-0.8,-0.7,0.4,1\}$. The speeds of the [1,4]- and [2,3]-solitons are given by $c_{14}=0.84$ and $c_{23}=0.37$. Consequently, the $[1,4]$-soliton moves further away from the $[2,3]$-soliton toward the positive $x$-axis and the nonlinear interaction between them fails to form the the other half of the $[1,4]$-soliton for $y>0$. The [1,4]-soliton looses energy as both its amplitude and slope decreases locally near the intersection point where it is transformed into a $[2,4]$-soliton which interacts resonantly with the top half of the $[2,3]$-soliton to generate a $[3,4]$-soliton for $y>0$. Thus, the intersection point becomes a three-wave resonant interaction vertex moving with velocity $v_{0}=(0.58,0.7)(\mathrm{cf}$. (8)). Therefore, it breaks off from the bottom half of the $[2,3]$-soliton which moves to the right and in the 
negative $y$-direction. The evolved wave-pattern is shown in Figure which also shows the decay of the bottom half of the $[2,3]$-soliton into dispersive waves after it detaches from the Y-soliton.

Yet another interesting situation occurs if we consider a half $[1,4]$-soliton for $y<0$ with a negative slope interacting with a full [2,3]-soliton with zero (or positive) slope as our initial condition. Here the faster moving [1,4]-half-soliton interacts nonlinearly as it passes through the [2,3]-soliton and a (4321)type soliton is formed near the intersection point which moves to the right and in the negative $y$-direction. The initial condition and its time evolution is displayed in Figure 15. After passing through the [2,3]soliton the leading edge of the $[1,4]$-soliton looses energy and slows down. Its amplitude decreases and the slope changes from negative to positive as it is transformed to a $[2,4]$-soliton as in the previous simulation in Figure 14. This $[2,4]$-soliton connects with the top half of the slowly propagating $[2,3]$-soliton to form a $[3,4]$-soliton for $y>0$ via three-wave resonant interaction. Thus the initial condition evolves into a (4321)-type soliton and a Y-soliton as shown in the right frame of Figure 15.
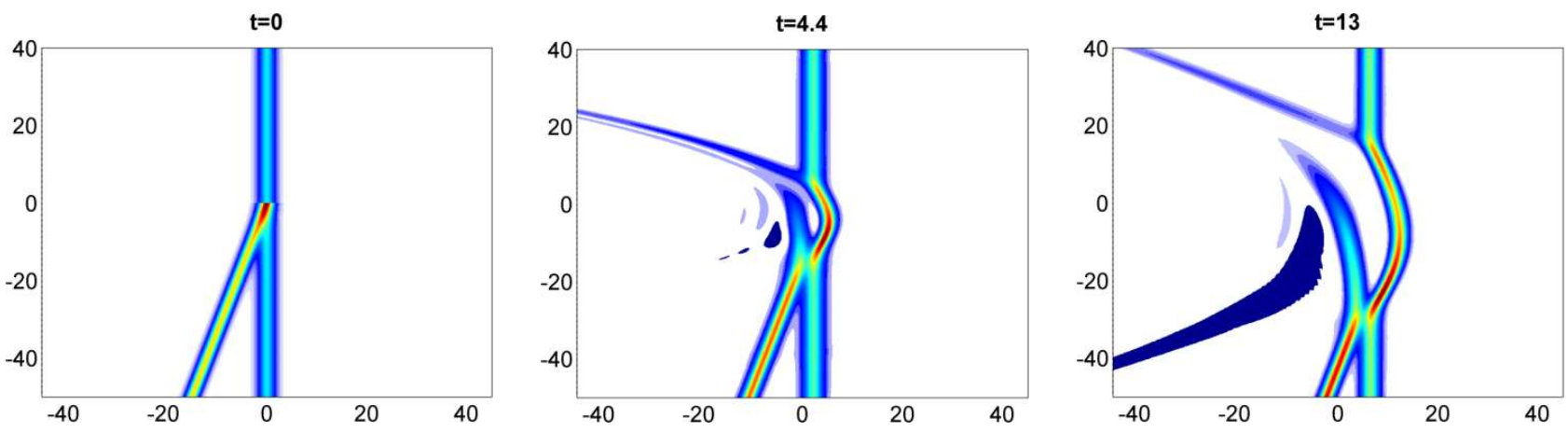

Figure 15: Non-convergence of initial data to a (4321)-type soliton.

\subsection{Bow-shape initial data}

In this subsection, we consider an initial condition obtained by adding a vertical stem of finite length to the <-shape, as shown in the top left frame of Figure 16. We call it the bow-shape initial data. Given a set of $k$-parameters $\left\{k_{1}, k_{2}, k_{3}, k_{4}\right\}$, we find that there are two distinct cases corresponding to whether the stem shrinks or grows. These cases are discussed below.

Case 1. (2413)-soliton: Here the bow-shape initial data is symmetric about the $x$-axis and consists of a $[1,2]$-soliton for $y \gg 0$, a $[3,4]$-soliton for $y \ll 0$, and a $[1,4]$-soliton as the initial stem. Specifically,

$$
u(x, y, 0)=\left\{\begin{array}{lc}
0.32 \operatorname{sech}^{2} 0.4(x-1.4 y+5.6), & y \geq 4, \\
2.42 \operatorname{sech}^{2} 1.1 x, & -4<y<4, \\
0.32 \operatorname{sech}^{2} 0.4(x+1.4 y+5.6), & y \leq-4,
\end{array}\right.
$$

with $\left\{k_{1}, k_{2}, k_{3}, k_{4}\right\}=\{-1.1,-0.3,0.3,1.1\}$. Note that the initial stem length is taken to be 8 units. The initial data and its time evolution are shown in the top panel of Figure 16. We observe that the stem in the initial bow shape shrinks with time by shedding energy in the form of dispersive radiation, which separates from the solitary waves. The shrinking of the stem can be explained by the KP theory in the following fashion. The top end of the stem is the intersection point of the [1,2]- and the [1,4]-solitons which are localized along the lines

$$
L_{12}: x+\left(k_{1}+k_{2}\right) y=c_{12} t+x_{12} \quad \text { and } \quad L_{14}: x+\left(k_{1}+k_{4}\right) y=c_{14} t+x_{14} .
$$



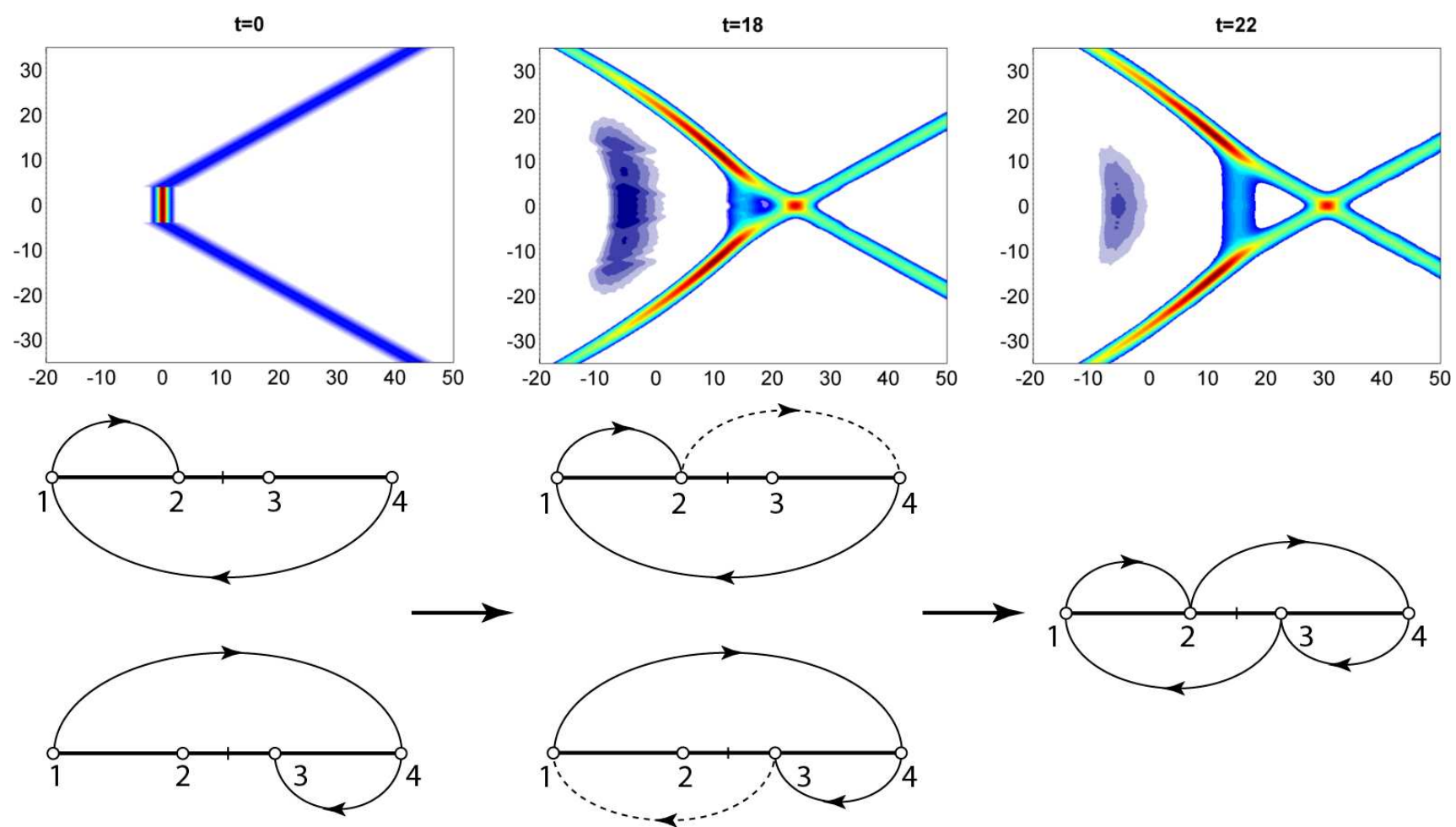

Figure 16: Top panel: Evolution of a bow-shape initial wave to a (2413)-type soliton. Domain $[-192,192] \times[-78,78]$. Initial stem length is 8 units. Bottom panel: Completion of the incomplete chords representing the bow-shape initial data. The dotted chords represent the newly generated $[2,4]$ and $[1,3]$-solitons, and the complete chord diagram represents a (2413)-type soliton.

Solving the above equations simultaneously, one obtains the coordinates $\left(x_{1}(t), y_{1}(t)\right)$ of the intersection point. In particular, $y_{1}(t)=\left(k_{1}+k_{2}+k_{4}\right) t+\left(x_{14}-x_{12}\right) /\left(k_{4}-k_{2}\right)$. Then, from the values of the $k$ parameters chosen above, $d y_{1} / d t=k_{1}+k_{2}+k_{4}<0$, implying the top end of the stem moves toward the $x$-axis. An analogous argument shows that the bottom end of the stem, which is the intersection point $\left(x_{2}(t), y_{2}(t)\right)$ of the [3,4]- and the [1,4]-solitons, also moves towards the $x$-axis as $y_{2}(t)=\left(k_{1}+k_{3}+k_{4}\right) t+$ $\left(x_{34}-x_{14}\right) /\left(k_{3}-k_{1}\right)$, so that $d y_{2} / d t>0$. Consequently, the length of the stem decreases.

The observed wave pattern corresponds to a (2413)-type (2,2)-soliton solution (see Figure 22), whose formation can be explained by the "gluing" of chords illustrated in the bottom panel of Figure 16. In this case the resonant interaction of the $[1,2]$ - and $[1,4]$-solitons create a $[2,4]$-soliton for $y<0$, while the $[3,4]$ and $[1,4]$-solitons create a $[1,3]$-soliton for $y>0$. These correspond to the two completed chords: one for the Y-shape (top) and the other for the $X$-shape (bottom). Next, by gluing these two chord diagrams and discarding the chords for the intermediate [2,3]-soliton, one obtains the (2413)-type chord diagram. Note that here the initial $[1,4]$-stem shrinks to a point where the $[1,2]$ - and $[3,4]$-solitons cross to make an Xshape, and a new small amplitude intermediate $[2,3]$-soliton is generated as shown in the right frame of the top panel of Figure 16. As the $[1,4]$-soliton shrinks, its energy transferred to the generation of the $[1,3]$ and $[2,4]$-solitons which are of higher amplitude than the $[1,2]$ - and $[3,4]$-solitons.

However, the wave pattern shown in Figure 16 is only a transient feature. The finite energy $[1,4]$ stem and the initial $[1,2]$ - and $[3,4]$-solitons do not have sufficient energy to sustain the formation of the $[1,3]$ - and $[2,4]$-solitons which are of higher amplitude. Continuing the simulation for a longer time, we observe that the amplitudes of the [1,3]- and [2,4]-solitons decrease until they transform into the $[1,2]$ and $[3,4]$-solitons, and the amplitude of the $[2,3]$-soliton decays. Finally, a steady wave pattern consisting only of an intersecting pair of [1,2]- and [3,4]-solitons is obtained. The latter corresponds to a (2143)-type solution of KP as shown in Figure 21. In order to verify our observation, we consider below another initial 
condition as above with a shorter stem. Specifically, we take

$$
u(x, y, 0)=\left\{\begin{array}{lc}
\frac{1}{2} \operatorname{sech}^{2} \frac{1}{2}(x-1.2 y+2.4), & y \geq 2, \\
2.42 \operatorname{sech}^{2} 1.1 x, & -2<y<2, \\
\frac{1}{2} \operatorname{sech}^{2} \frac{1}{2}(x+1.2 y+2.4), & y \leq-2,
\end{array}\right.
$$

with $\left\{k_{1}, k_{2}, k_{3}, k_{4}\right\}=\{-1.1,-0.1,0.1,1.1\}$, and the initial stem length is now 4 units. The initial data and its time evolution are shown in the top panel of Figure 17. In this case, the (2413)-type wave form
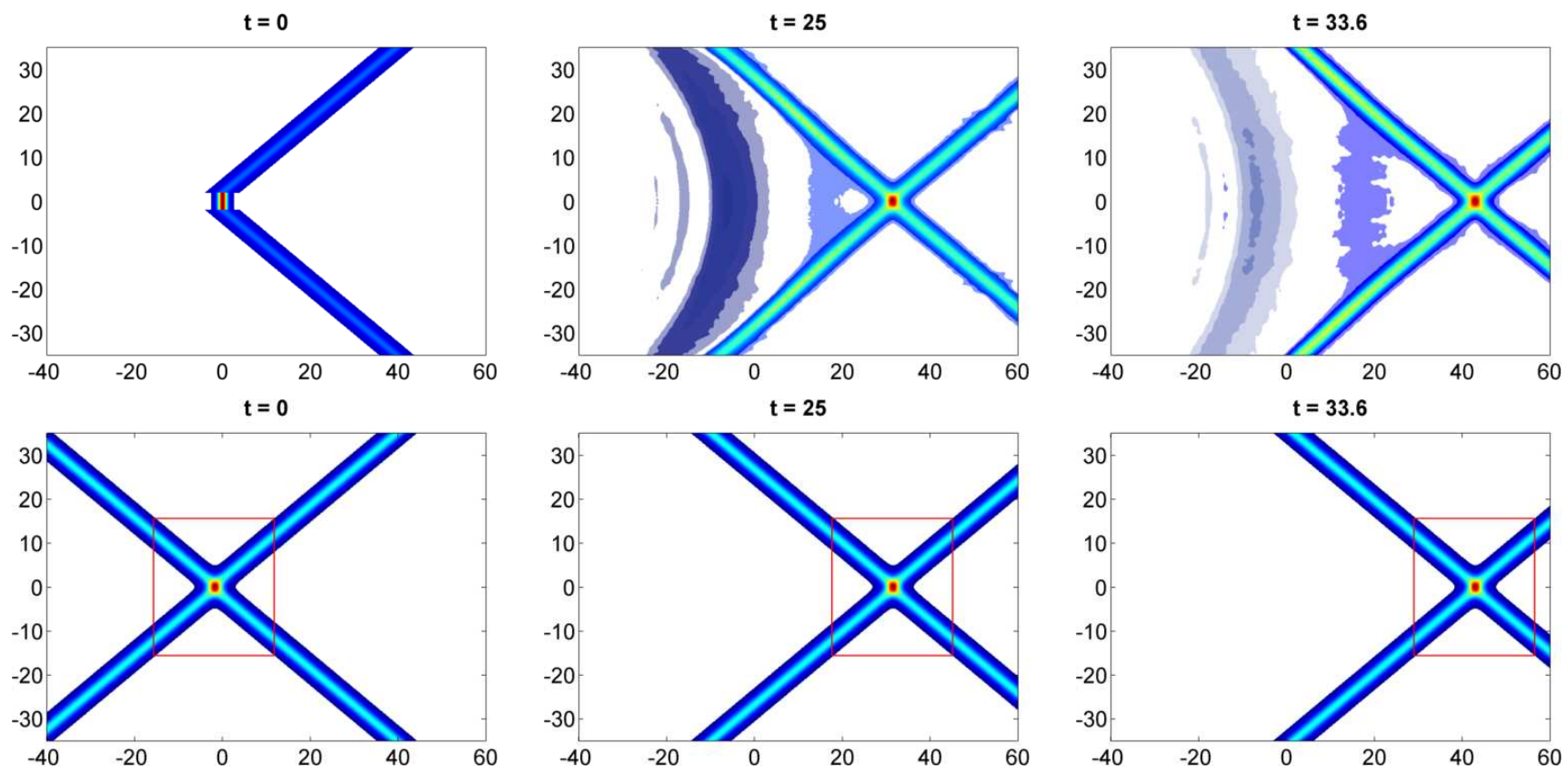

Figure 17: Convergence of a bow-shape initial wave to a (2143)-type soliton. Initial stem length is 4 units. Domain $[-200,200] \times[-78,78]$.

dissipates, and a stable (2143)-type solution with an X-shape pattern emerges. The only remnant of the transient (2413)-type pattern is the low amplitude, intermediate [2,3]-soliton which is slowly decaying as seen in the middle and right frames. In this case the partial chord diagram and its completion are the same as that of a <-shape initial condition since the [1,4]-stem decays, and consequently, does not represent a soliton. The associated chord diagrams are shown in the left and middle frames of Figure 6. We confirm the formation of the (2143)-type solution by comparing the numerical solution with an exact solution with optimal parameter values which minimize the error $E(t)$. We take a box size of $27.5 \times 31$, enclosing the interaction region near the shrinking stem (which eventually becomes the vertex of the Xshape) for the minimization problem. The box center given by $x_{0}(t)=1.21 t, y_{0}(t)=0$ is the intersection point of the $[1,4]$-stem with the $x$-axis. The optimal values of the parameters for the exact solution are $x_{12}^{+}=x_{34}^{+}=-2.334$ corresponding to the $x$-intercepts at $t=0$ for the [1,2]- and [3,4]-solitons for $x>0$. Then from Table 1 (Appendix A), one can construct the $A$-matrix to be

$$
A=\left(\begin{array}{cccc}
1 & 18.915 & 0 & 0 \\
0 & 0 & 1 & 1.723
\end{array}\right)
$$

The resulting (2143)-type solution is shown in the bottom panel of Figure 17. The error plot on the left frame of Figure 18 shows convergence of the wave pattern to the exact solution. We needed to extend our numerical domain in $x$ to $[-200,200]$ for this simulation, since the local $\mathrm{X}$-shape pattern moves close to the right-hand boundary for run times close to $t=40$. The right frame in Figure 18 shows the evolution of 

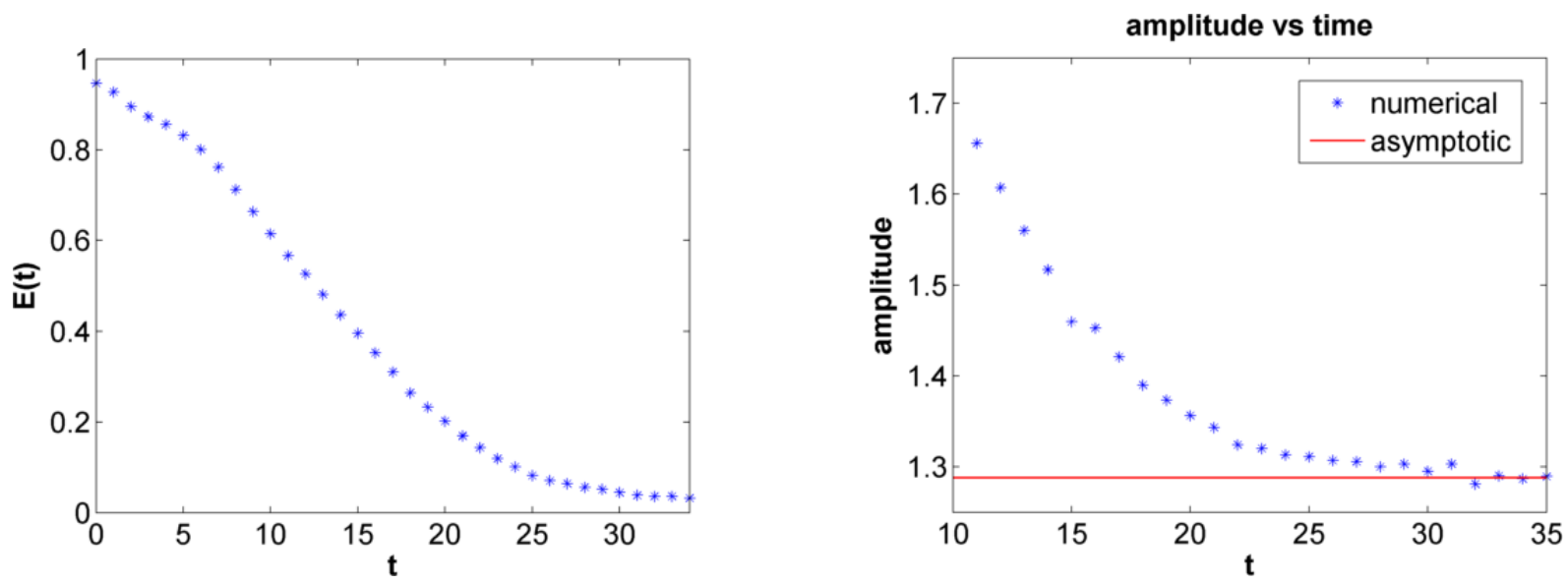

Figure 18: Convergence of a bow-shape initial data to a (2143)-type soliton. Left: Error $E(t)$ vs time, error minimized at $t=24.2$. Right: Amplitude $u_{\max }$ at the intersection point of the $\mathrm{X}$-shape.

the peak amplitude of the stem at $y=0$. As the stem shrinks, the peak amplitude decreases and converges to the theoretically predicted value of the maximum amplitude for the exact (2143)-type (2,2)-soliton solution at its $\mathrm{X}$-vertex. This behavior provides further evidence that the evolving wave pattern converges to a (2143)-type KP soliton. The theoretical estimate for the maximum amplitude at the X-vertex of the (2143)-type solution is given by [7]

$$
u_{\max }=A_{1}+A_{2}+2 \sqrt{A_{1} A_{2}}\left(\frac{1-\sqrt{\Delta}}{1+\sqrt{\Delta}}\right), \quad \Delta=\frac{\left(k_{3}-k_{2}\right)\left(k_{4}-k_{1}\right)}{\left(k_{4}-k_{2}\right)\left(k_{3}-k_{1}\right)},
$$

where $A_{1}=\frac{1}{2}\left(k_{2}-k_{1}\right)^{2}, A_{2}=\frac{1}{2}\left(k_{4}-k_{3}\right)^{2}$ are the amplitudes of the [1,2] and [3,4] solitons, respectively. Using the chosen $k$-values, we obtain $u_{\max }=1.288$, which is what our numerically estimated value approaches asymptotically in time.

Case 2. (3142)-soliton: Next we consider a bow-shape initial data

$$
u(x, y, 0)=\left\{\begin{array}{lc}
\frac{1}{2} \operatorname{sech}^{2} \frac{1}{2}(x-0.8 y+1.6), & y \geq 2, \\
1.62 \operatorname{sech}^{2} 0.9 x, & -2<y<2, \\
\frac{1}{2} \operatorname{sech}^{2} \frac{1}{2}(x+0.8 y+1.6), & y \leq-2,
\end{array}\right.
$$

which consists of a $[1,3]$-soliton for $y>0$, a $[2,4]$-soliton for $y<0$, and a $[1,4]$-soliton as the initial stem of length 4 units. The $k$-parameters are chosen as $\left\{k_{1}, k_{2}, k_{3}, k_{4}\right\}=\{-0.9,-0.1,0.1,0.9\}$. The initial data and its time evolution are shown in the top panel of Figure 19. By comparing the frames at $t=6$ and $t=22$, it is clear that the stem length of the bow-shape grows in this case. This growth can also be explained by the KP theory in a similar fashion as in Case 1 above. In this case, the completion of chords and the gluing procedure leads to a (3142)-type chord diagram as shown in the bottom panel of Figure 19. For $y>0$, the $[1,3]$ - and the [1,4]-soliton interact resonantly to form a [3,4]-soliton for $x<0$, behind the [1,3]-soliton. Similarly for $y<0$, the $[2,4]$ - and the $[1,4]$-soliton interact resonantly to form a $[1,2]$-soliton for $x<0$, behind the $[2,4]$-soliton. Then the gluing of the two chord diagrams yield the (3142)-type soliton.

One can also verify by measuring the amplitudes and slopes of the line solitons that the initial wave form converges to a (3142)-type soliton after separating from the dispersive waves. The error, $E(t)$, between the numerical and the exact solutions is shown at the bottom panel of Figure 19. The error was minimized at $t=22$ by optimizing the parameters $x_{14}, x_{13}$, and $x_{24}$ corresponding to the exact (3142)-type soliton solution (see Table 1, Appendix A) displayed in the middle panel of the figure. The optimized 

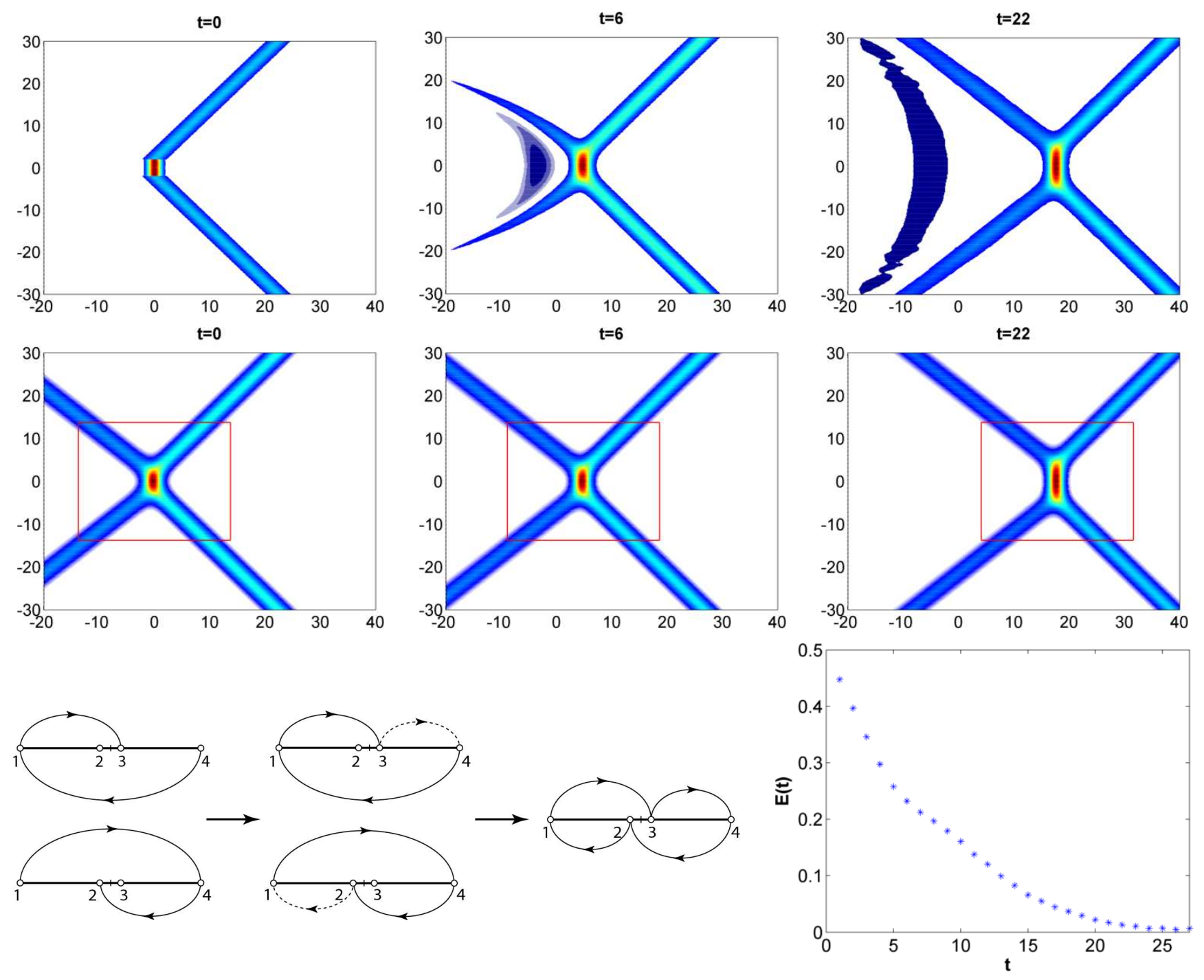

Figure 19: Top panel: Evolution of a bow-shape initial wave to a (3142)-soliton. Domain $[-192,192] \times$ $[-78,78]$. Initial stem length is 4 units. Middle panel: Evolution of the exact solution with optimized $A$-matrix. Bottom panel: Completion of the incomplete chords representing the initial data to a (3142)soliton (left frame). $E(t)$ vs $t$ plot (right frame). Error minimized at $t=22$.

values of the parameters are $x_{14}=-0.232$ and $x_{13}=x_{24}=-1.613$. From these optimized values of the $x_{i j}$, the $A$-matrix (see Table 1 ) is calculated to be

$$
A=\left(\begin{array}{cccc}
1 & 1.513 & 0 & -1.898 \\
0 & 0 & 1 & 0.168
\end{array}\right)
$$

The box size used for the minimization of the error was $27.5 \times 27.5$, and its center $x_{0}(t)=0.81 t, y_{0}(t)=0$ corresponds to the intersection of the $[1,4]$-soliton with the $x$-axis. The error decreases with $t$, showing convergence to the exact (3142)-type solution.

Behavior of the stem: In our simulations, we observe that the stem initially loses energy to radiative dispersion, and its shape and amplitude fluctuate considerably due to interaction with dispersive waves. This behavior contrasts the smooth evolution of the stem for the (3142)-type soliton with only a $<$-shape initial data without the stem part as shown in [8, 12]. After this initial phase, the stem starts to form into an intermediate $[1,4]$-soliton due to the resonant interactions with the other asymptotic line-solitons. The left frame in the top panel of Figure 20 shows the numerical estimate (asterisks) of the stem amplitude 
averaged around the center $(y=0)$ of the stem as well as the amplitude at $y=0$ (circles) of the intermediate $[1,4]$-soliton of the exact (3142)-type solution. The numerical estimate starts at a value of 1.62 at $t=0$, then sharply drops before increasing (from below) toward the asymptotic value of $\frac{1}{2}\left(k_{4}-k_{1}\right)^{2}=1.62$. The stem amplitude for the exact solution increases monotonically from an initial value of 1.28 , and for $t>30$, it almost coincides with the numerically estimated values. This plot illustrates the transformation of the initial stem into an intermediate $[1,4]$-soliton described by the KP theory. For comparison, we have also included the numerical data (cross) for the stem amplitude corresponding to a $<$-shape initial data with the same $k$-values as above. In this case the maximum amplitude at $y=0$ grows from 0.5 (amplitude of the initial <-shape wave form) to about 1.26 when $\mathrm{t}=10$, and continues to grow but at a much slower rate so that it takes longer time $(t>45)$ before it gets close to the stem amplitude of the exact solution (circles). This suggests that the energy from the finite stem in the bow-shape initial data accelerates the growth of the intermediate $[1,4]$-soliton of the (3142)-type solution.
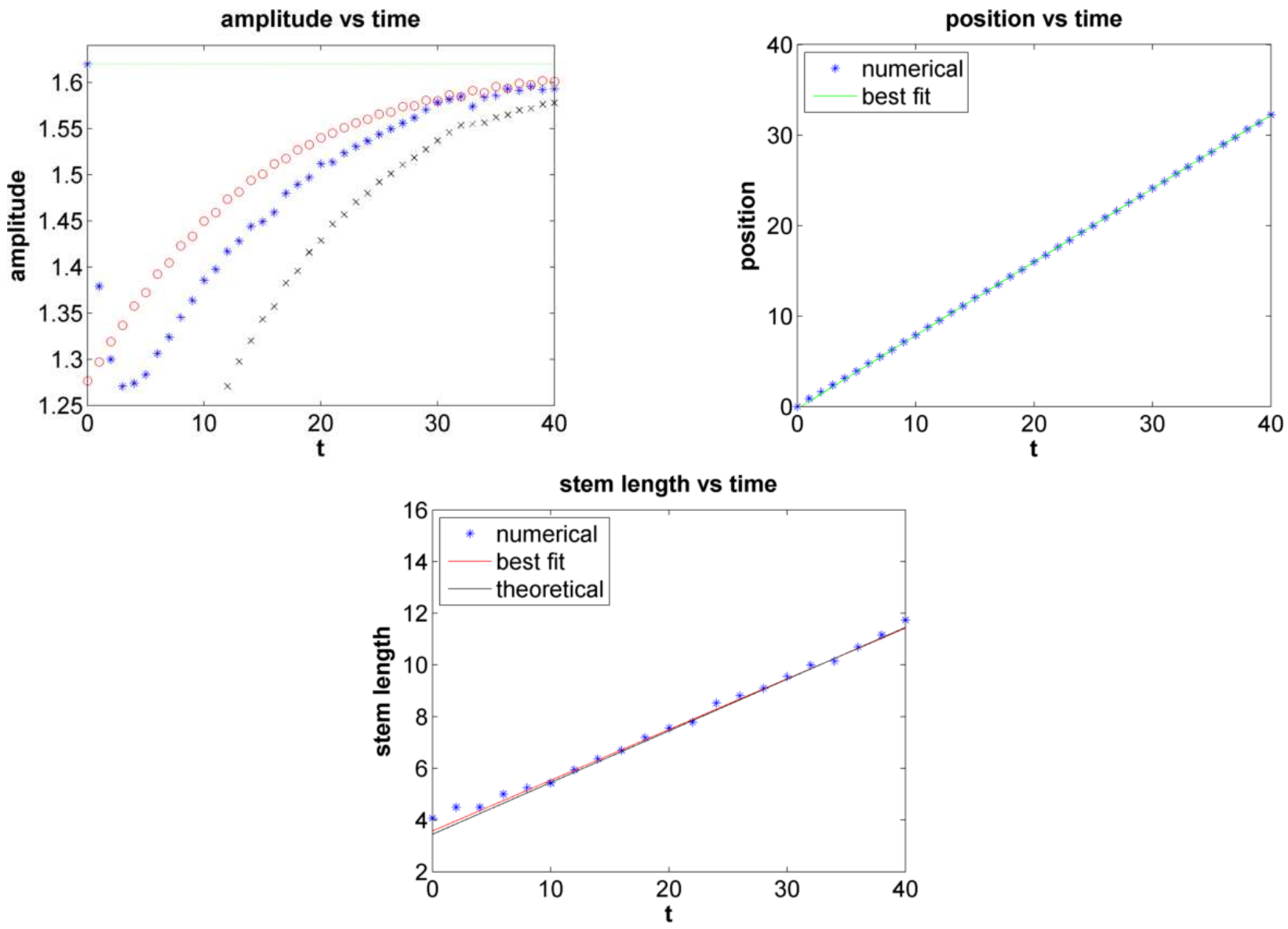

Figure 20: Stem dynamics for a bow-shape initial condition. Top left: growth of stem amplitude. Circles - amplitude of exact solution at $y=0$; asterisks - amplitude of numerical solution averaged around $y=0$; cross - amplitude at $y=0$ of a numerical solution evolving from a $<$-shape initial condition. Top right: evolution of the stem center for the numerical solution. Bottom: Plot of stem length vs time.

The right frame on the top panel of Figure 20 shows the evolution of the center of the stem at $y=0$. The slope $(=0.808)$ of the best-fit line to this curve agrees with the theoretical value of the stem velocity $c_{14}=k_{1}^{2}+k_{1} k_{4}+k_{4}^{2}=0.81$. From the intercept of the best-fit line, we find $x_{14}=-0.247$ which is in good agreement with the optimized value of $x_{14}=-0.232$. The negative position shift of the $[1,4]$-soliton is owing to its loss of momentum due to the formation of the [1,2]- and [3,4]-solitons for $x<0$.

The bottom panel of Figure 20 shows the numerically estimated growth of the stem length versus time. The length of the stem increases as the $[1,4]$-soliton is formed. Note that from around $t=5$ or so, the stem 
starts to grow almost linearly with time. The linear growth can be explained from the KP theory. The top and bottom ends of the stem are given by the intersection of the [1,4]-soliton with the [1,3]-soliton and the $[2,4]$-soliton, respectively. Using (8), one finds that

$$
\Delta y(t)=y_{1}(t)-y_{2}(t)=\left(k_{3}-k_{2}\right) t+\frac{x_{14}-x_{13}}{k_{4}-k_{3}}+\frac{x_{14}-x_{24}}{k_{2}-k_{1}},
$$

where the $y$-coordinates of the top and bottom ends of the stem are denoted by $y_{1}(t)$ and $y_{2}(t)$, respectively. For this case, the theoretical growth for the stem length is given by $\Delta y(t)=0.2 t+3.453$ after inserting the values of the parameters used. For our numerical estimate, we performed a linear interpolation of the numerical data from $t=10$ to $t=40$. The equation of the best fit-line through the numerical data is $0.196 t+3.581$, which is in good agreement with the theoretical estimate.

\section{Conclusion}

In this paper we have conducted a numerical study of the initial value problem for the KP equation using specific types of initial data that converge to the $(2,2)$-soliton solutions. Our numerical simulations demonstrate that the piece-wise line solitons in the initial waveform interact to create dispersive waves and local spatial shifts propagating along the soliton wavecrests away from the interaction region. The waveform in the interaction region converges to a KP soliton, and the shifts along the wave crests lead to nonzero position shifts $x_{i j}$ of the asymptotic solitons of the exact solution. The convergence of the numerical solution is in a local sense over a compact region in the $x y$-plane and is measured by the $\mathrm{L}^{2}$ error between the numerical and exact solution over this region. Our numerical simulations support the notion of the minimal completion of the partial chord diagrams representing the initial data [12]. Thus, the classification of KP solitons via fixed-point free permutations [5, 7] play a crucial role in our investigations. We have provided examples to illustrate how the completion of chords depend on additional constraints that facilitate nonlinear interactions depending on the speed and direction of propagation of the initial waveforms. In our examples, we have also analyzed the nonlinear interactions which often result in the formation of intermediate solitons due to the three-wave resonant interaction process. We have studied the dynamics of the stems and compared our numerical results to the KP theory. Our results demonstrate that at least, to leading order, the numerical wave patterns can be approximated by the KP solitons over any compact region inside of the numerical domain.

To the best of our knowledge, there have not been any significant work on the numerical study of the KP initial value problem except for the ones reported in $[12,13,8]$ which considered special types of initial conditions. In comparison, our work presents new numerical simulations with a much broader class of initial conditions based on the combinatorial ideas of chord completion and gluing of intermediate chord diagrams (see e,g., [6]).

In future work we plan to study more complex wave patterns containing a large number of asymptotic solitons associated with more general $(M-N, N)$-soliton solutions of the KP equation. We hope that such studies will shed more light on the question of stability of the the KP solitons. Moreover, investigations of the initial data for more general types of KP solitons will provide further evidence for the chord completion conjecture and perhaps sufficient information to prove (or disprove) this conjecture.

\section{Acknowledgments}

This research was partially supported by NSF under grant numbers DMS-1108694 \& DMS-1410862 (SC) and DMS-1410267 (YK). The authors thank Y. Jia for a preliminary version of MATLAB code. 

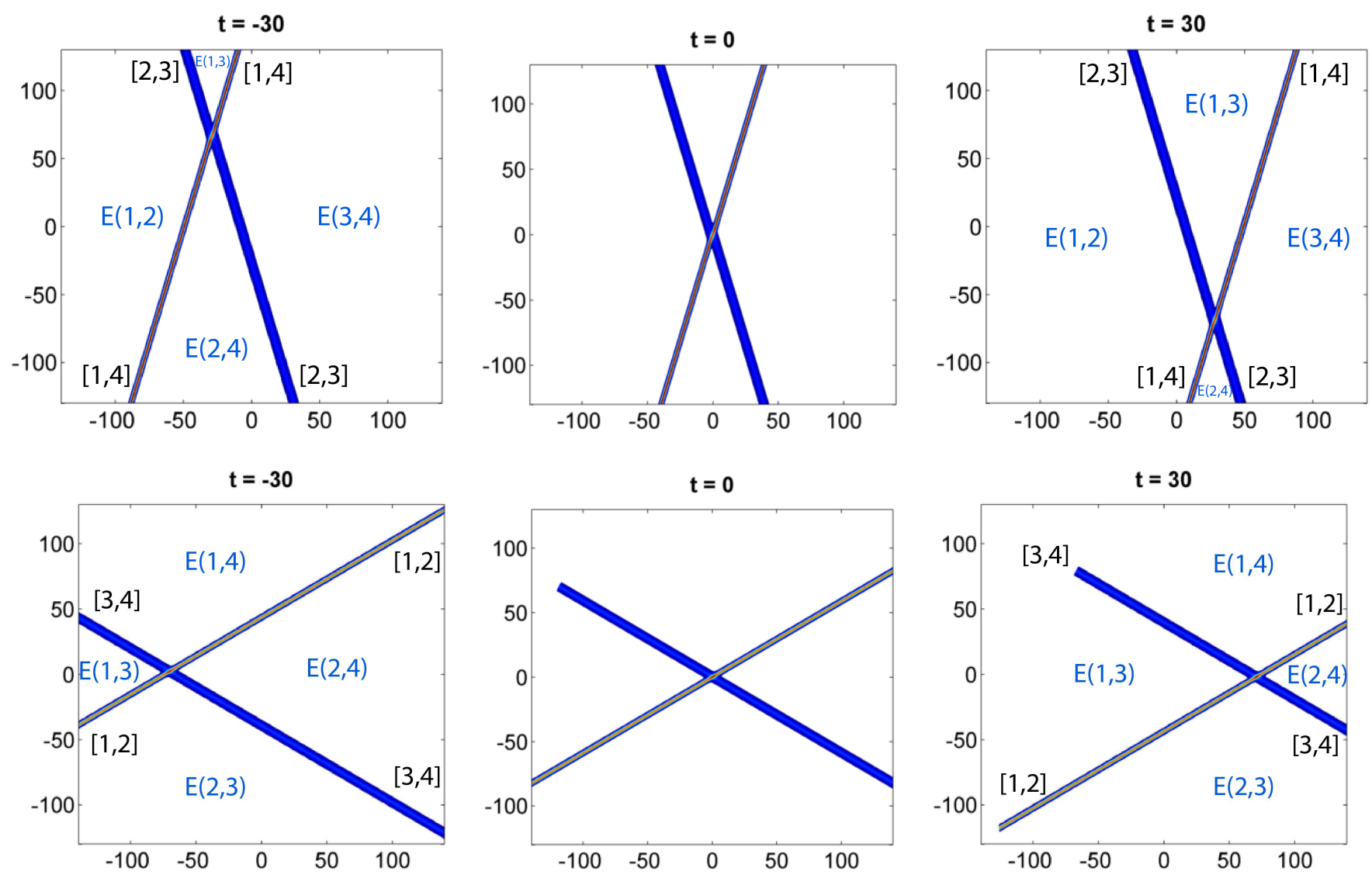

Figure 21: Top panel: A (4321)-type and bottom panel: a (2143)-type (2,2)-soliton solution of the KP equation with $k$-parameters: $\{-1.4,-0.3,0.6,1.1\}$.

\section{Appendix}

\section{A Summary of (2,2)-solitons}

In this appendix, we summarize the essential features of all seven types of (2,2)-soliton solutions. Six of the seven (2,2)-soliton solutions are plotted in Figures 21 and 22. (The plot of the (3412)-type soliton solution was given earlier in Figure 5). Each panel shows a (2,2)-soliton for $t<0, t=0$, and $t>0$ with the same values of the $k$-parameters $\left\{k_{1}, k_{2}, k_{3}, k_{4}\right\}=\{-1.4,-0.3,0.6,1.1\}$. For each solution, the parameters in the $A$-matrix are chosen (see Table 1 below) such that all the asymptotic solitons meet at the origin in the $x y$-plane at $t=0$. This configuration can be achieved by setting the $x$-intercept $x_{i j}=0$ for each asymptotic $[i, j]$-soliton. Then the corresponding $A$-matrix can be read off from Table 1 . The asymptotic and the intermediate solitons as well as the dominant exponential functions in each region of the $x y$-plane are indicated in the figures. As the solution evolves in time, different intermediate solitons are formed due to resonant interactions, whereas the asymptotic solitons as $|y| \gg 0$ remain invariant with respect to time.

In Figure 22 the (4312)- and the (3421)-type solitons shown in the top two panels are dual to each other in the sense that one type of solution is transformed to the other by the transformation $(x, y, t) \rightarrow$ $(-x,-y,-t)$. Moreover, their chord diagrams in Figure 4 are also related to each other by a reflection about the horizontal axis switching the upper and lower pair of chords. This means that the the asymptotic solitons for these two types of solutions as $y \gg 0$ and as $y \ll 0$ are switched, as seen from Figure 22 . Similarly, the (2413)- and the (3142)- types of (2,2)-solitons shown in the the third and fourth panels 
of Figure 21 form another set of dual pair. On the other hand, the (3412)-type solution in Figure 5, the (4321)- and (2143)-type solutions shown in Figure 21 are self-dual. That is, these solutions are invariant under the discrete transformation $(x, y, t) \rightarrow(-x,-y,-t)$, so that they have the same pair of asymptotic solitons as $y \rightarrow \pm \infty$. Consequently, their chord diagrams admit a reflection symmetry about the horizontal axis as shown in Figure 4.

Table 1 below lists the corresponding $A$-matrix for each type of (2,2)-soliton, as well as expressions relating the $A$-matrix parameters and the $x$-intercepts $x_{i j}$ of a subset of the asymptotic and intermediate solitons. Note that these relations are not unique since each element of the $A$-matrix can be expressed via the $x_{i j}$ of more than one subset of $[i, j]$-solitons in a given $(2,2)$-soliton solution.

Table 1: The seven types of $(2,2)$-soliton solutions of the KP equation. Notations: $|j, i|:=k_{j}-k_{i}$, $\theta_{i j}:=\left(k_{j}-k_{i}\right) x_{i j}, i<j$. The superscripts $\theta_{i j}^{ \pm}$denote the $[i, j]$-soliton for $y>0$ or $y<0$, and $\theta_{14}^{*}$ in the first row denotes the intermediate $[1,4]$-soliton for $t<0$. See Figure 5.

\begin{tabular}{|c|c|c|}
\hline $\begin{array}{c}\text { Type } \\
(\pi)\end{array}$ & $A$-matrix & $A$-matrix parameters \\
\hline$(3412)$ & $\begin{array}{c}\left(\begin{array}{cccc}1 & 0 & -c & -d \\
0 & 1 & a & b\end{array}\right), \\
\quad a, b, c, d>0 \\
D:=a d-b c>0\end{array}$ & $\begin{array}{ll}b=\frac{|2,1|}{|4,1|} e^{-\theta_{24}^{+}}, & c=\frac{|2,1|}{|3,2|} e^{-\theta_{13}^{-},} \\
\frac{D}{b}=\frac{|4,1|}{|4,3|} e^{-\theta_{13}^{+}}, & d=\frac{|2,1|}{|4,2|} e^{-\theta_{14}^{*}}\end{array}$ \\
\hline$(4312)$ & $\begin{array}{c}\left(\begin{array}{cccc}1 & 0 & -b & -c \\
0 & 1 & a & 0\end{array}\right) \\
a, b, c>0\end{array}$ & $\begin{array}{c}b=\frac{|2,1|}{|3,2|} e^{-\theta_{13}}, \quad c=\frac{|3,1|}{|4,3|} e^{-\theta_{14}}, \\
\frac{a c}{b}=\frac{|3,2|}{|4,3|} e^{-\theta_{24}}\end{array}$ \\
\hline$(3421)$ & $\begin{array}{c}\left(\begin{array}{cccc}1 & 0 & 0 & -c \\
0 & 1 & a & b\end{array}\right) \\
a, b, c>0\end{array}$ & $\begin{array}{c}b=\frac{|2,1|}{|4,1|} e^{-\theta_{24}}, \quad c=\frac{|3,1|}{|4,3|} e^{-\theta_{14}}, \\
\frac{a c}{b}=\frac{|4,1|}{|4,3|} e^{-\theta_{13}}\end{array}$ \\
\hline$(2413)$ & $\begin{array}{c}\left(\begin{array}{cccc}1 & 0 & -c & -d \\
0 & 1 & a & b\end{array}\right) \\
a, b, c, d>0 \\
a d-b c=0\end{array}$ & $\begin{array}{c}b=\frac{|2,1|}{|4,1|} e^{-\theta_{24}}, \quad c=\frac{|2,1|}{|3,2|} e^{-\theta_{13}}, \\
\frac{d}{b}=\frac{|4,1|}{|4,2|} e^{-\theta_{12}}\end{array}$ \\
\hline$(3142)$ & $\begin{array}{c}\left(\begin{array}{cccc}1 & a & 0 & -c \\
0 & 0 & 1 & b\end{array}\right), \\
a, b, c>0\end{array}$ & $\begin{array}{l}s=e^{-\theta_{14}}, \quad a=\frac{|3,1|}{|3,2|} s e^{\theta_{24}}, \\
b=\frac{|3,1|}{|4,1|} s e^{\theta_{13}}, \quad c=\frac{|3,1|}{|4,3|} s\end{array}$ \\
\hline$(4321)$ & $\begin{array}{c}\left(\begin{array}{cccc}1 & 0 & 0 & -b \\
0 & 1 & a & 0\end{array}\right), \\
a, b>0\end{array}$ & $a=\frac{|4,2|}{|4,3|} e^{-\theta_{23}^{-}}, \quad b=\frac{|3,1|}{|4,3|} e^{-\theta_{14}^{+}}$ \\
\hline$(2143)$ & $\begin{array}{c}\left(\begin{array}{llll}1 & a & 0 & 0 \\
0 & 0 & 1 & b\end{array}\right), \\
a, b>0\end{array}$ & $a=\frac{|4,1|}{|4,2|} e^{-\theta_{12}^{+}}, \quad b=\frac{|3,2|}{|4,2|} e^{-\theta_{34}^{-}}$ \\
\hline
\end{tabular}



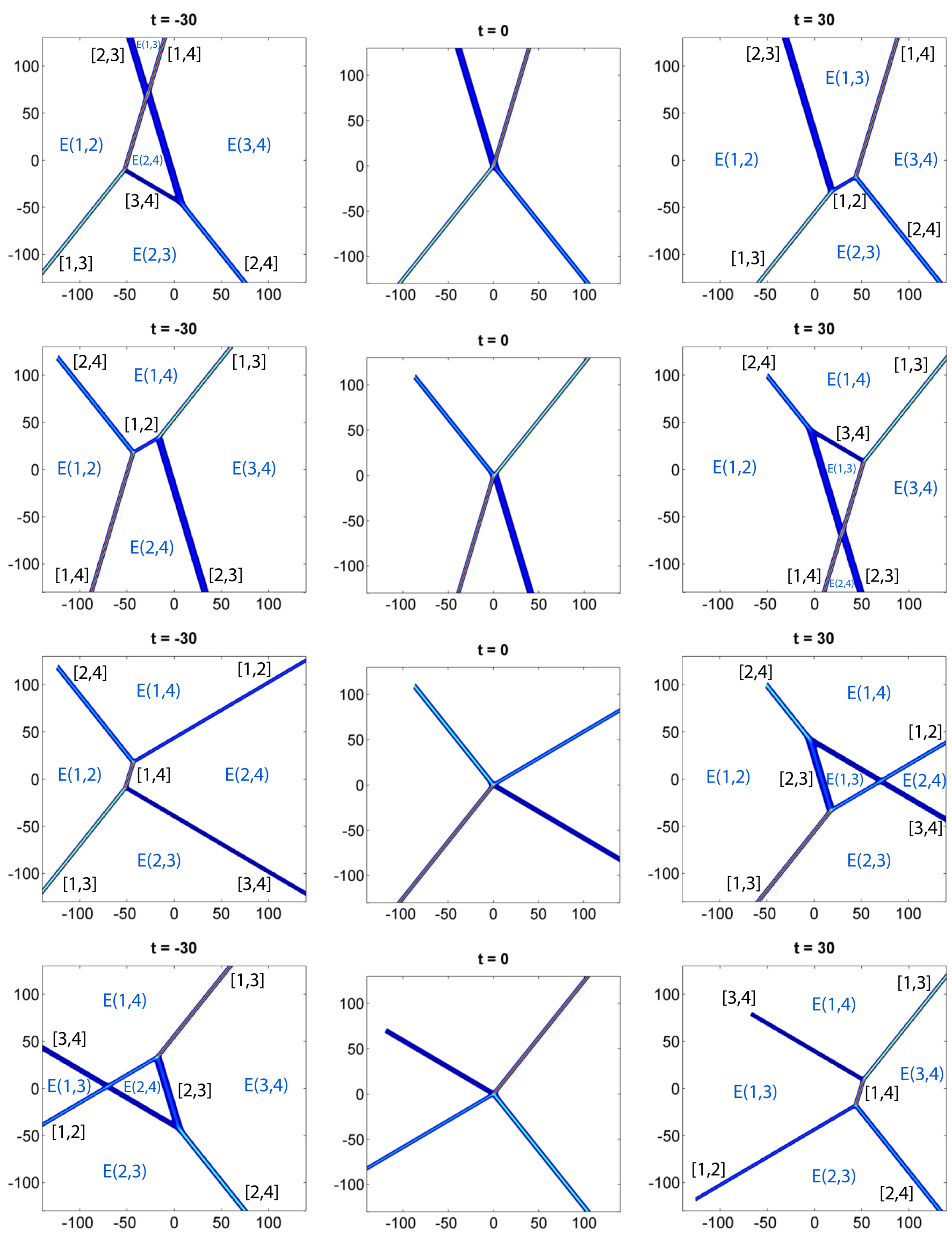

Figure 22: Panels 1 - 4 are plots of (4312), (3421), (2413) and (3142) types of (2,2)-soliton solutions. The $k$-parameters are the same as in Figure 21. 


\section{B Numerical scheme \& accuracy}

In this appendix we describe the numerical scheme used to perform the simulations presented in Section 3. This scheme was first implemented in the numerical simulations presented by some of the current authors in [8] primarily for <-shape initial data. In this paper, we use the same numerical scheme which is included below for sake of completeness. Furthermore, we include a brief discussion of the numerical tests we performed to verify the scheme accuracy using $L^{2}$ error estimates of the numerical versus the exact solution of the KP equation.

\section{B.1 Numerical Scheme}

We solve the KP equation numerically using a pseudo-spectral scheme on a rectangular domain $D=$ $\left\{(x, y):|x| \leq \frac{1}{2} L_{x},|y| \leq \frac{1}{2} L_{y}\right\}$. We assume that the solution is periodic in both $x$ and $y$. It is then convenient to rescale $D$ to a fixed domain $D^{\prime}=\{(X, Y):|X| \leq \pi,|Y| \leq \pi\}$ by defining $X=\left(2 \pi / L_{x}\right) x$ and $Y=\left(2 \pi / L_{y}\right) y$ so that (1) becomes

$$
\left(u_{t}+P u u_{X}+Q u_{3 X}\right)_{X}+R u_{2 Y}=0, \quad P=\frac{3 \pi}{L_{x}}, Q=\frac{2 \pi^{3}}{L_{x}^{3}}, R=\frac{3 \pi L_{x}}{2 L_{y}^{2}} .
$$

Due to the periodicity assumption, the solution can be expressed as a Fourier series

$$
u(X, Y, t)=\sum_{l=-\infty}^{\infty} \sum_{m=-\infty}^{\infty} \hat{u}(l, m, t) e^{i(l X+m Y)}
$$

and reduce (12) into an ODE for the time evolution of the Fourier coefficients $\hat{u}(l, m, t)$, namely,

$$
\hat{u}_{t}+\frac{i l P}{2} \mathcal{N}(\hat{u})+i\left(\frac{R m^{2}}{l}-Q l^{3}\right) \hat{u}=0, \quad l \neq 0,
$$

where $\mathcal{N}(\hat{u})$ is the Fourier transform of $u^{2}$ which is numerically evaluated as $\left.\mathcal{N}(\hat{u})=\operatorname{FFT}\left[(\operatorname{IFFT}(\hat{u}))^{2}\right)\right]$. The ODE (13) can be expressed as

$$
\hat{v}_{t}+\alpha e^{c t} \mathcal{N}\left(\hat{v} e^{-c t}\right)=0,
$$

where $c=i\left(R m^{2} / l-Q l^{3}\right), \hat{v}=\hat{u} e^{c t}$, and $\alpha=i l P / 2$. It is then numerically solved using the Runge-Kutta (RK4) method for a given initial data $\hat{u}(l, m, 0)$ obtained from the Fourier Transform of $u(X, Y, 0)$. The solution $u(X, Y, t)$, and hence $u(x, y, t)$, is then reconstructed by taking the inverse Fourier Transform of $\hat{u}(l, m, t)$.

When $l=0,(13)$ requires that $\hat{u}(0, m, t)=0$ for all values of $m$ and $t$. This is called the zero-mode condition, which is equivalent to the mean-free condition: $\int_{-\pi}^{\pi} u d X=0$ and must be satisfied for all periodic solutions. However, our initial data (in the form of line solitons) do not satisfy the mean-free condition. In our numerical code, we force the zero-modes at any given time to be the same as the initial value i.e., we set $\hat{u}(0, m, t)=\hat{u}(0, m, 0)$. Consequently, our scheme generates some error. However, numerical tests (see Appendix B.2) suggest that the maximum error in the Fourier domain introduced due to the violation of the zero-mode condition remains small (see also [13]).

The pseudo-spectral scheme works well for periodic boundary conditions in both $x$ and $y$ directions. The initial data used in our numerical simulations are in the form of oblique solitary waves that are rapidly decreasing in the $x$-direction. Choosing $L_{x}$ of the numerical domain to be large enough guarantees (almost) vanishing boundary condition, and consequently, periodicity in the $x$-direction. However, since the initial data is not periodic in the $y$-direction, and non-vanishing at $y= \pm \frac{1}{2} L_{y}$, reflected waves are generated from 
the boundary and affect the numerical domain. Furthermore, the amplitude of the wave near the boundary diminishes, and the wave crest is bent backward due to dispersive waves created at the boundary. To address these issues, we use a method developed by Tanaka [16] (see also [17]) to artificially modify the data near the boundary to maintain constant amplitude and unperturbed shape. For the types of initial data used in our numerical simulations, we modify the numerical solution by imposing zero boundary conditions in the $x$-direction and by "patching" with the exact one-soliton solutions in the $y$-direction near the boundary. That is, the solution in the patching region evolves according to the $[i, j]$-soliton prescribed in (5), whereas the solution inside the patching region evolves according to the numerical scheme. Figure 23 below shows an example of a $\mathrm{V}$-shape initial condition with the indicated patching region at the boundary. The patching region is chosen to be $L_{x} / 8$ on the left and right, and $L_{y} / 8$ on top and bottom of the rectangular domain.

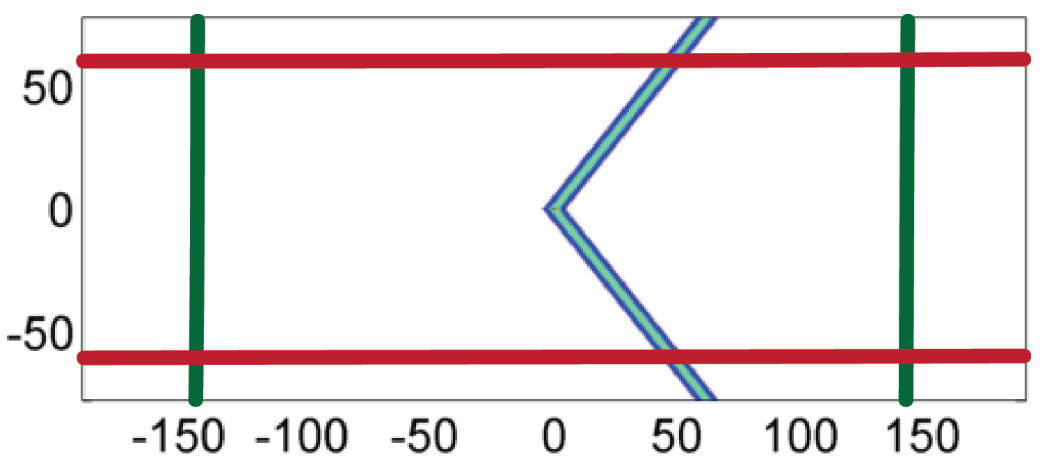

Figure 23: A V-shape initial condition with patching

\section{B.2 Scheme accuracy}

We perform several numerical tests to check the accuracy of our numerical scheme. These tests also help us select optimal values for the number of Fourier modes per unit length in both $x$ and $y$ for the FFT computation as well as the optimal time-step $\Delta t$ in the RK4, in our numerical scheme. For these tests, we perform numerical simulations of the (3412)-type KP soliton with an X-shape initial data as shown in Figure 5 (middle frame) using our numerical scheme and compare with the corresponding exact solution. Note that our initial data for the simulations are chosen to be the same as the exact solutions at $t=0$. The $k$-parameter values for the initial condition are chosen to be $\left\{k_{1}, k_{2}, k_{3}, k_{4}\right\}=\{-1,-0.25,0.25,1\}$. For these simulations, we take the same number of Fourier modes per unit length in both $x$ and $y$, and the spatial domain $D$ is chosen to be $\left[-\frac{1}{2} L_{x}, \frac{1}{2} L_{x}\right] \times\left[-\frac{1}{2} L_{y}, \frac{1}{2} L_{y}\right]=[-128,128] \times[-64,64]$.

At first we estimate the error as a function of number of Fourier modes per unit length. To run these simulations, we take the time step $\Delta t=0.005$ for the RK4 integration. Figure 24 shows both the absolute error and the relative error (in $\log$ scale) defined as $E_{\text {abs }}:=\left\|u_{\text {num }}-u_{\text {exact }} \mid\right\|_{D}$, and $E_{\text {rel }}:=E_{\text {abs }} /\left|u_{\text {exact }}\right| \|_{D}$ with respect to the number of Fourier modes $n$ per unit length $t=30$. Here, $\|\cdot\|_{D}$ denotes the $\mathrm{L}^{2}$ norm over domain $D$. Notice that the absolute error over the domain $\mathrm{D}$ is $\approx 10^{-2}$ and the relative error is less than $10^{-4}$ when $n=12$, i.e., using 3072 and 1536 Fourier modes in $x$ and $y$, respectively. However, running the simulations with such a fine mesh over large computational domains takes a long time. Additionally, in our numerical experiments we typically use a larger domain than used here in order to account for the patched region.

Next, we examine the accuracy of our scheme with respect to the time-step $\Delta t$ for the numerical integration using RK4. For these simulations, we take the number of Fourier modes per unit length $n=8$ in both $x$ and $y$ directions and run up to $t=30$ using values of $\Delta t=0.08,0.04,0.02,0.01$, and 0.005 . The left panel in Figure 25 below shows the relative error $E_{\text {rel }}$ for different values of $\Delta t$ at $t=2, t=15.2$ and 

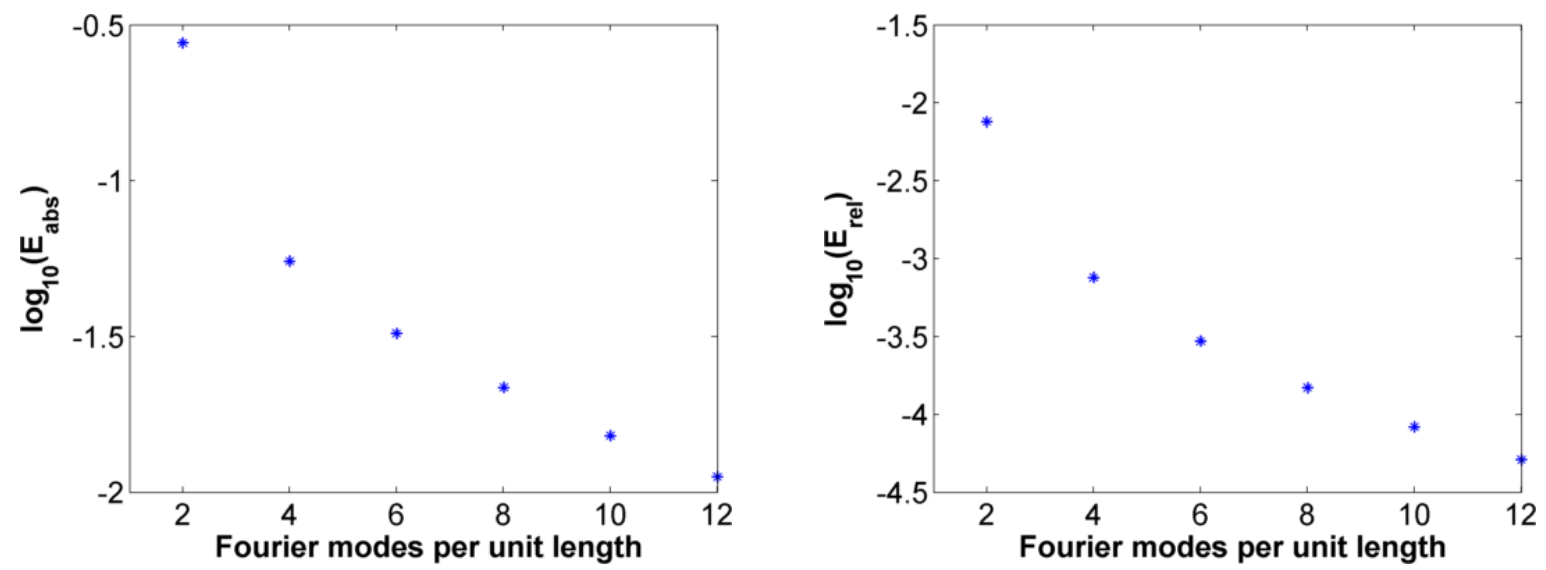

Figure 24: Numerical accuracy for a (3412)-type solution. Plots of $E_{\mathrm{abs}}$ and $E_{\mathrm{rel}}$ vs the number of Fourier modes per unit length at $t=30$. Numerical Domain $D=[-128,128] \times[-64,64]$.

$t=30$. We find that the relative error initially decreases and then saturates for small time steps. The value of $E_{\text {rel }}$ can be decreased further if we increase the the number of Fourier modes from $n=8$, but again the computational time increases significantly.
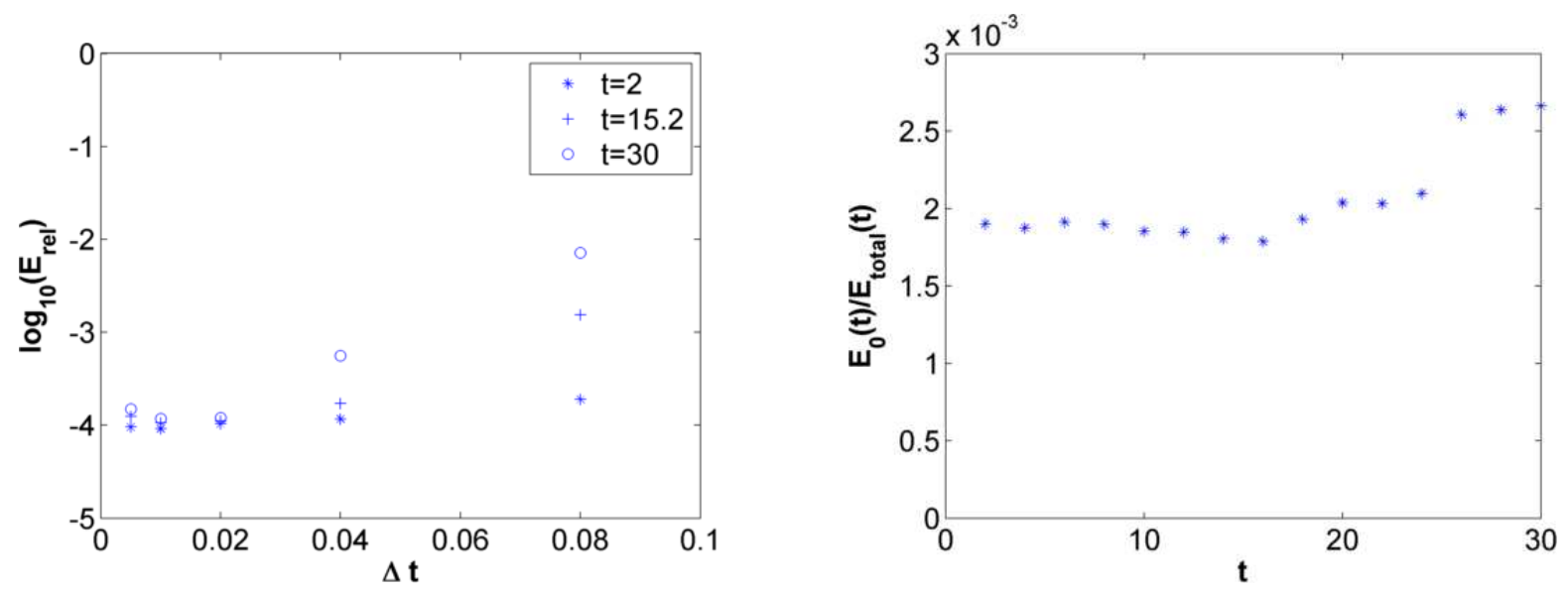

Figure 25: Left panel: Plot of $E_{\text {rel }}$ vs time step $\Delta t$ at $t=2,15.2,30$. Number of Fourier modes per unit length $n=8$. Numerical Domain $D=[-128,128] \times[-64,64]$. Right panel: Growth of the $l=0$ Fourier mode in the numerical simulation of a (3412)-type soliton.

In most of our numerical simulations, we take the entire numerical domain to be $[-192,192]$ in $x$ and $[-78,78]$ in $y$. The unpatched region is $[-144,144]$ in $x$ and $[-58.5,58.5]$ in $y$. In order to strike a balance between accuracy and speed based on the above tests, we choose 8 Fourier modes per unit length in both $x$ and $y$ directions for the FFT calculations and a time step of $\Delta t=0.005$ for the RK4 scheme. With these optimal values, the absolute error and the relative errors are always within $2.5 \times 10^{-2}$ and $1.8 \times 10^{-4}$, respectively, for a typical run up to $t=35$ in all our numerical simulations discussed in Section 3.

Finally, we address the issue of the error caused by the zero-mode described in Section B.1. Since we force $\hat{u}(0, m, t)=\hat{u}(0, m, 0)$ at each time step, we can estimate the induced error due to the growth of the zero modes by comparing with the exact solution. The right frame in Figure 25 illustrate the growth of the zero mode for the (3412)-type solution from our numerical simulations on a $[-128,128] \times[-64,64]$ spatial domain with $n=8$ Fourier modes per unit length in $x$ and $y$, and time step $\Delta t=0.005$. It plots the ratio $E_{0}(t) / E_{\text {tot }}(t)$ as a function of time, where $E_{0}(t):=\sum_{m}\left|\hat{u}_{\text {num }}(0, m, t)-\hat{u}_{\text {exact }}(0, m, t)\right|$ is the $L^{1}$ error in the $l=0$ Fourier mode and $E_{\text {tot }}:=\sum_{l, m}\left|\hat{u}_{\text {num }}(l, m, t)-\hat{u}_{\text {exact }}(l, m, t)\right|$ is the total error in the Fourier domain 
summed over all the Fourier modes. This plot suggests that although the zero-mode error grows over time, its overall contribution to the total numerical error remains small, i.e., less that $0.3 \%$ of the total error $E_{\text {tot }}$ over a run time of up to $t=30$. Consequently, the accuracy of our numerical scheme is not compromised by the zero-mode error.

\section{References}

[1] M. J. Ablowitz and H. Segur, Solitons and the inverse scattering transform, (SIAM, Philadelphia, 1981).

[2] B. B. Kadomtsev and V. I. Petviashvili, On the stability of solitary waves in weakly dispersing media, Sov. Phys. Doklady 15, 539-541 (1970)

[3] M. J. Ablowitz and D. E. Baldwin, Nonlinear shallow ocean-wave soliton interactions on flat beaches, Phys. Rev. E 86 (2012) 036305

[4] G. Biondini and S. Chakravarty, Soliton solutions of the Kadomtsev-Petviashvili II equation, J. Math. Phys. 47 (2006) 033514.

[5] S. Chakravarty and Y. Kodama, Classification of the line-solitons of KPII, J. Phys. A: Math. Theor. 41 (2008) 275209 (33pp).

[6] S. Chakravarty and Y. Kodama, Construction of KP solitons from wave patterns, J. Phys. A: Math. Theor. 47 (2014) 025201 (17pp)

[7] S. Chakravarty and Y. Kodama, Soliton solutions of the KP equation and application to shallow water waves, Stud. Appl. Math. 123 (2009) 83-151.

[8] S. Chakravarty, T. McDowell, M. Osborne, Numerical studies of the KP line-solitons, Commun Nonlinear Sci Numer Simulat 44 (2017) 37-51.

[9] W. Li, H. Yeh and Y. Kodama, On the Mach reflection of a solitary wave: revisited, J. Fluid Mech. 672 (2011) pp. 326-357.

[10] Y. Kodama, Young diagrams and $N$-soliton solutions of the KP equation, J. Phys. A: Math. Gen., 37 (2004) 11169-90.

[11] Y. Kodama, KP solitons in shallow water, J. Phys. A: Math. Theor., 43 (2010) 434004 (54pp).

[12] Y. Kodama, M. Oikawa and H. Tsuji, Soliton solutions of the KP equation with V-shape initial waves, J. Phys. A: Math. Theor., 42 (2009) 312001 (9pp).

[13] C-Y Kao and Y. Kodama, Numerical study of the KP equation for non-periodic waves, Math. Comput. Simul. 82 (2012) 1185-1218.

[14] Y. Kodama and L. Williams, KP solitons and total positivity for the Grassmannian, Invent. Math. 195 (2014)

[15] Y. Kodama and H. Yeh, The KP theory and Mach reflection, J. Fluid. Mech., 800 (2016) 766-786.

[16] M. Tanaka, Mach reflection of a large-amplitude solitary waves, J. Fluid Mech., 248 (1993) 637-661.

[17] Y. Jia, Numerical study of the KP solitons and higher order Miles theory of the Mach reflection in shallow water, Ph.D. Thesis, Ohio State University (2014). 